\title{
Thermodynamic Analysis of Isobutane Dehydrogenation Reaction
}

\author{
Mazhar Abdulwahed \\ College of Sciences, University of Damascus, Damascus, Syria \\ E-mail: m.abdulwahed@damasuniv.edu.sy
}

Received 18 January 2015, Revised 08 July 2015, Accepted 09 July 2015

\begin{abstract}
The thermodynamics of isobutane dehydrogenation reaction was investigated thoroughly using most reliable literature data. Conversion and reaction mixture distribution at equilibrium were computed for various process conditions. Reaction enthalpy, $\Delta_{R} H_{T}^{o}$, ranges between $122 \pm 1.1$ and $123 \pm 1.2 \mathrm{~kJ} . \mathrm{mol}^{-1}$ over the temperature 773.15 to $923.15 \mathrm{~K}$ where the equilibrium constant $K_{T}$ value varies between $0.12 \pm 0.03$ and $2.6 \pm 0.7$. The reaction becomes thermodynamically favorable at temperatures higher than $873.15 \mathrm{~K}$.
\end{abstract}

Keywords: Isobutane; 2-methylpropane; dehydrogenation; thermodynamic analysis.

\section{Introduction}

Dehydrogenation reactions are largely employed in the chemical industry. The route to obtain principally olefins, for example, isobutene, is generally carried out using isobutane dehydrogenation on $\mathrm{Cr}$ - or Pt-based catalysts. $\mathrm{iC}_{4} \mathrm{H}_{10}(\mathrm{~g})=$ $\mathrm{iC}_{4} \mathrm{H}_{8}(\mathrm{~g})+\mathrm{H}_{2}(\mathrm{~g})$ Open literature lacks however technically convenient information about thermodynamic equilibrium description of this reaction.

The Catofin ${ }^{\circledR}$ and the Snamprogetti-Yarsintezi ${ }^{\circledR}$-FBD4 are the only two commercially proven dehydrogenation processes using Cr-based catalysts. Both processes do not use diluent to the feed gas. Catofin ${ }^{\circledR}$ process operates at subatmospheric pressure in the temperature range 860 to $920 \mathrm{~K}$. While the FBD4 process operates at slightly higher than atmospheric pressure in the temperature range 800 to $860 \mathrm{~K}$ [1-3]. The Oleflex ${ }^{\circledR}$ process is the sole commercial process based on platinum catalyst, it operates at slightly higher than atmospheric pressure using feed gas enriched with hydrogen under moderate temperatures $[2,4]$.

Literature described a variety of methods for prediction of thermodynamic properties of isobutane, isobutene and hydrogen. Therefore, wide discrepancy exists among experimental and estimated values of equilibrium constants and conversions [5-12].

In this work it is attempted to estimate thermodynamic parameters, including molar enthalpy, molar Gibbs free energy, equilibrium constant, conversion and product distribution of isobutane dehydrogenation reaction. Emphasis will be given to industrially relevant conditions.

\section{Computational Work}

\subsection{Criteria for Input Data Selection}

The main difficulty in analyzing thermodynamic equilibrium of reactions is to evaluate the Gibbs free energy, $\Delta_{R} G_{T}^{o}$. As $\pm 1 \mathrm{~kJ}^{-\mathrm{mol}^{-1}}$ uncertainty in Gibbs free energy amplifies to $\pm 12 \%$ uncertainty in equilibrium constant at $1000 \mathrm{~K}$ due to exponential dependency between both parameters [13a, 14].

Differences in estimated or measured quantities of standard enthalpy of formation $\Delta H_{f}^{o}$, standard free enthalpy of formation (Gibbs free energy of formation) $\Delta G_{f}^{o}$, standard entropy of formation $S_{i}^{o}$, and molar gas phase heat capacity $C_{p}^{o}$ (contributors to $\Delta_{R} G_{T}^{o}$ ) are frequently at the core of disputes among groups of researchers.

Accuracy of thermodynamic quantities usually differs following to the technique of measurement, or following to the method of estimation. For example, $\Delta H_{f}^{o}$ usually measured by bomb or flame calorimetric techniques. Both techniques are trustworthy [14], however each yields own accuracy. On the other hand, estimation methods exhibit dissimilar accuracies [6-9,12,15]. The thermodynamic quantity should be therefore defined with sufficient completeness with respect to the required accuracy so that all practical purposes associated with this quantity is unique. The usefulness of thermodynamic data, and thus much of the information that it provides, is therefore largely determined by the quality of the statements of uncertainty. To meet the needs of some industrial and commercial applications expanded uncertainty $U_{e}$ is moreover favored [16]. Hence, both uncertainty information will be provided throughout this work.

The first part of this work will be devoted to evaluate available experimental thermodynamic data involved in calculation. Estimated thermodynamic quantities will be used, whenever experimental values are missing.

Best available experimental quantity $(x)$ will be selected based on relative standard uncertainty $u_{r}(x)$, which is defined as the absolute difference $\Delta x$ between measured and a reference quantity $x_{\text {calc. }}$ divided by the reference quantity: $u_{r}(x)=\frac{|\Delta x|}{x_{\text {calc. }}}$, whereby $x_{\text {calc. }}$ is a "conventional true value" of the described thermodynamic quantity estimated favorably by Benson's method [12,13a,17] or through its improvement by Domalski and Hearing [7]. Exceptions will be indicated if any. This criteria is expected to act as uniform uncertainty information of thermodynamic quantities in literature. Calculation is then proceeded as follow. 


\subsection{Calculation Method}

The following gives the summary of calculations:

$\Delta_{R} H_{298.15}^{o}=\sum v_{i} \Delta H_{f, i}^{o}$

$\Delta_{R} S_{298.15}^{o}=\sum v_{i} S_{f, i}^{o}$

$\Delta_{R} G_{298.15}^{o}=\sum v_{i} \Delta G_{f, i}^{o}$

$\ln K_{T}=\frac{-\Delta_{R} G_{T}^{o}}{R T}=\frac{-\Delta_{R} H_{T}^{o}}{R T}+\frac{\Delta_{R} S_{T}^{o}}{R}$

Both $\Delta_{R} H_{T}^{o}$ and $\Delta_{R} S_{T}^{o}$ are temperature dependent according to Eqs. (5) and (6).

$$
\begin{aligned}
& \Delta_{R} H_{T}^{o}=\Delta_{R} H_{298.15}^{o}+\int_{298.15}^{T} \Delta_{R} C_{p_{T}^{o}}^{o} \mathrm{dT} \\
& \Delta_{R} S_{T}^{o}=\Delta_{R} S_{298.15}^{o}+\int_{298.15}^{T} \frac{\Delta_{R} C_{p_{T}^{o}}^{o}}{T} \mathrm{dT}
\end{aligned}
$$

The heat capacity of reaction mixture can be defined as follows:

$\Delta_{R} C_{p_{T}^{o}}^{o}=\sum v_{i} C_{p_{T}, i}^{o}$

The values of individual molar heat capacities $C_{p_{T}, i}^{o}$ follow diverse temperature functions. Thus, the results of Eq. (5) or (6) differ accordingly.

Temperature dependency of the heat capacities of gases is commonly represented by polynomials of the form $[13 b, 18,19]$ :

$C_{p_{T}, i}^{o}=a_{o, i} T+a_{1, i / 2} T^{2}+a_{2, i / 3} T^{3}+a_{3, i / 4} T^{4}+\ldots$

Poling et. al [13b], Reid et. al [18] and Kyle [20] give a set of thermodynamically consistent polynomials of the order four or five for estimation the ideal gas heat capacity. These polynomials functions will be critically evaluated in next section. Shomate function specified in [21], and other functions given in [22] and [19] were excluded due to less properly fitting to existing experimental data.

Thus, using Eqs. (7) and (8), it is now possible to compensate for temperature-induced changes in both the enthalpy and entropy terms respectively in Eqs. (5) and (6), allowing the calculation of thermodynamic equilibrium constants. This is accomplished by substitution and rearrangement that yields the followings:

$\Delta_{R} C_{p_{T}^{o}}^{o}=\Delta a_{o} T+\frac{\Delta a_{1}}{2} T^{2}+\frac{\Delta a_{2}}{3} T^{3}+\frac{\Delta a_{3}}{4} T^{4}$

whereby: $\Delta a_{n}=\Delta_{R} a_{n}=\sum v_{i} a_{n, i}$ $n: 0,1,2$ or 3 and $i$ : reaction species

$\ln K_{T}=\left[\frac{-\Delta_{R} H_{298.15}^{o}}{T}+\Delta_{R} S_{298.15}^{o}+\Delta a_{o}\left(\ln \frac{T}{298.15}+\right.\right.$ $\left.\left(\frac{298.15}{T}-1\right)\right)+\Delta a_{1}\left((T-298.15)-\frac{1}{2 T}\left(T^{2}-\right.\right.$

$\left.\left.298.15^{2}\right)\right)+\Delta a_{2}\left(\frac{1}{2}\left(T^{2}-298.15^{2}\right)-\frac{1}{3 T}\left(T^{3}-298.15^{3}\right)\right)+$ $\left.\Delta a_{3}\left(\frac{1}{3}\left(T^{3}-298.15^{3}\right)-\frac{1}{4 T}\left(T^{4}-298.15^{4}\right)\right)\right] \frac{1}{R}$

It is also possible to compute free reaction enthalpy at temperature $T$ from Eq. (4) or from Eq. (11):

$\Delta_{R} G_{T}^{o}=\Delta_{R} H_{T}^{o}-T \Delta_{R} S_{T}^{o}$
The relationship between $K$ and equilibrium conversion $X_{(i C 4 H 10, e)}$ can be determined using the following path:

$K=\frac{\boldsymbol{y}_{i C 4 H 8} \cdot \boldsymbol{y}_{H 2}}{\boldsymbol{y}_{i C 4 H 10}}\left(\frac{P^{o}}{P}\right)^{-v}$

where $v$ is the reaction stoichiometric coefficients

$v=\sum v_{i}$

Mole fraction can be expressed by using reaction number $\varepsilon$ :

$y_{i}=\frac{\dot{n}_{i o}+v_{i} \varepsilon}{\dot{n}_{o}+v \varepsilon}$

$\dot{n}_{o}=\sum_{i}^{\text {feed }} n_{\text {lo }}$

$=$ sum of mole streams of all feed components $\left(\mathrm{iC}_{4} \mathrm{H}_{10}\right.$, and $\mathrm{H}_{2}$, inert gas if any)

$\varepsilon=\dot{n}_{i C 4 H 10, o} . X$

Thus, using Eqs. (12)-(16), it is possible to express the relationship between the $K$ and $X$ :

$K=\frac{X(h+X)}{(1+h+i+X)(1-X)} P$

where $i$ and $h$ are:

$i=\frac{\dot{n}_{\text {inert }}}{\dot{n}_{i C 4 H 10, o}}, h=\frac{\dot{n}_{H 2}}{\dot{n}_{i C 4 H 10, o}}$

$i$ and $h$ are the number of moles of inert gases and hydrogen per mole of isobutane in the feed, respectively.

In some industrial operations, like in Oleflex process, part of the hydrogen produced is recycled to inhibit the formation of coke on the catalyst. However this is not the case with the other two commercial processes. Thus, for inert/hydrogen free isobutane feed the equilibrium conversion can be readily calculated by

$K=\frac{X^{2}}{1-X^{2}} P$

\subsection{Heat Capacity Regression Coefficients}

Regression of several mathematical polynomials for gas phase heat capacity was compared with experimental data from literature. Regression coefficients of these polynomials for related reaction species are given in Tables $1-3$. Evaluation criteria is described in section 2.1.

Table 1. Gas Phase Heat Capacity Regression Coefficients for Isobutane, over the T-range 298-1000K.

\begin{tabular}{cll}
\hline Coefficients & Poling et. al [13b] & Reid et. al [18] \\
\hline$a_{0, i}$ & 27.860214 & -1.39 \\
$a_{1, i}$ & 0.148679262 & $3.85 \mathrm{E}-01$ \\
$a_{2, i}$ & 0.000455358 & $-1.85 \mathrm{E}-04$ \\
$a_{3, i}$ & $-6.73 \mathrm{E}-07$ & $2.90 \mathrm{E}-08$ \\
$a_{4, i}$ & $2.69 \mathrm{E}-10$ & \\
\hline
\end{tabular}

Table 2. Gas Phase Heat Capacity Regression Coefficients for Isobutene, over the T-range 298-1000K.

\begin{tabular}{cll}
\hline coefficients & Poling et. al [13b] & Reid et. al [18] \\
\hline$a_{0, i}$ & 26.862534 & $1.61 \mathrm{E}+01$ \\
$a_{1, i}$ & $1.74 \mathrm{E}-01$ & $2.80 \mathrm{E}-01$ \\
$a_{2, i}$ & 0.000192303 & $-1.09 \mathrm{E}-04$ \\
$a_{3, i}$ & $-3.2832 \mathrm{E}-07$ & $9.10 \mathrm{E}-09$ \\
$a_{4, i}$ & $1.30197 \mathrm{E}-10$ & \\
\hline
\end{tabular}


Table 3. Gas Phase Heat Capacity Regression Coefficients for Hydrogen, over the T-range 298-1000K.

\begin{tabular}{llll}
\hline coefficients & $\begin{array}{l}\text { Poling et. al } \\
{[13 \mathrm{~b}]}\end{array}$ & $\begin{array}{l}\text { Reid et. al } \\
{[18]}\end{array}$ & Kyle [20] \\
\hline$a_{0, i}$ & 23.969262 & 27.14 & 29.11 \\
$a_{1, i}$ & 0.030603834 & $9.274 \mathrm{E}-03$ & $-1.92 \mathrm{E}-03$ \\
$a_{2, i}$ & $-6.4184 \mathrm{E}-05$ & $-1.381 \mathrm{E}-05$ & $4.00 \mathrm{E}-06$ \\
$a_{3, i}$ & $5.75329 \mathrm{E}-08$ & $7.645 \mathrm{E}-09$ & $-8.70 \mathrm{E}-10$ \\
$a_{4, i}$ & $-1.7709 \mathrm{E}-11$ & & \\
\hline
\end{tabular}

Tables 4 - 6 compare heat capacities estimated using these polynomials with experimental data. Due to absence of experimental data for isobutene, estimated values from Kilpatrick and Pitzer [15] have been used.

Table 4. Experimental and Estimated Gas Phase Heat Capacities $C_{p}$ of Isobutane between 298.15 and $1000 \mathrm{~K}$.

\begin{tabular}{cc|cc|cc}
\hline$T / \mathrm{K}$ & $\begin{array}{c}\text { Exp. } C_{p} \\
{[23]}\end{array}$ & $\begin{array}{c}\text { Est. } C_{p} \\
{[18]}\end{array}$ & $u_{r}\left(C_{p}\right) \times 100$ & $\begin{array}{c}\text { Est. } C_{p} \\
{[13 \mathrm{~b}]}\end{array}$ & $u_{r}\left(C_{p}\right) \times 100$ \\
\hline 298 & 96.56 & 97.67 & 1.15 & 96.95 & 0.41 \\
300 & 97.06 & 98.19 & 1.16 & 97.45 & 0.40 \\
500 & 149.10 & 148.43 & 0.45 & 148.71 & 0.26 \\
$\mathbf{7 0 0}$ & $\mathbf{1 8 8 . 2 8}$ & $\mathbf{1 8 7 . 3 8}$ & $\mathbf{0 . 4 8}$ & 188.78 & 0.26 \\
$\mathbf{8 0 0}$ & $\mathbf{2 0 3 . 6 4}$ & $\mathbf{2 0 3 . 0 5}$ & $\mathbf{0 . 2 9}$ & 203.81 & 0.08 \\
$\mathbf{9 0 0}$ & $\mathbf{2 1 6 . 9 4}$ & $\mathbf{2 1 6 . 4 2}$ & $\mathbf{0 . 2 4}$ & 216.37 & 0.26 \\
$\mathbf{1 0 0 0}$ & $\mathbf{2 2 8 . 4 5}$ & $\mathbf{2 2 7 . 6 6}$ & $\mathbf{0 . 3 5}$ & 227.92 & 0.23 \\
\hline
\end{tabular}

Table 5. Experimental and Estimated Gas Phase Heat Capacities $C_{p}$ Isobutene between 298.15 and $1000 \mathrm{~K}$.

\begin{tabular}{cc|cc|cc}
\hline$T / \mathrm{K}$ & $\begin{array}{c}\text { Est. } C_{p} \\
{[15]}\end{array}$ & $\begin{array}{c}\text { Est. } C_{p} \\
{[18]}\end{array}$ & $u_{r}\left(C_{p}\right) \times 100$ & $\begin{array}{c}\text { Est. } C_{p} \\
{[13 \mathrm{~b}]}\end{array}$ & $u_{r}\left(C_{p}\right) \times 100$ \\
\hline 298.15 & 89.12 & 90.16 & 1.17 & 88.18 & 1.05 \\
300 & 89.5 & 90.60 & 1.23 & 88.61 & 0.99 \\
400 & 111.17 & 111.34 & 0.15 & 109.62 & 1.39 \\
500 & 130.71 & 130.11 & 0.46 & 129.12 & 1.22 \\
600 & 147.70 & 146.98 & 0.49 & 146.55 & 0.78 \\
$\mathbf{7 0 0}$ & $\mathbf{1 6 2 . 3 8}$ & $\mathbf{1 6 1 . 9 9}$ & $\mathbf{0 . 2 4}$ & 161.66 & 0.45 \\
$\mathbf{8 0 0}$ & $\mathbf{1 7 5 . 1 4}$ & $\mathbf{1 7 5 . 2 0}$ & $\mathbf{0 . 0 4}$ & 174.50 & 0.36 \\
$\mathbf{9 0 0}$ & $\mathbf{1 8 6 . 3 1}$ & $\mathbf{1 8 6 . 6 7}$ & $\mathbf{0 . 1 9}$ & 185.46 & 0.46 \\
$\mathbf{1 0 0 0}$ & $\mathbf{1 9 6 . 0 2}$ & $\mathbf{1 9 6 . 4 5}$ & $\mathbf{0 . 2 2}$ & 195.21 & 0.41 \\
\hline
\end{tabular}

Table 6. Experimental and Estimated Gas Phase Heat Capacities $C_{p}$ of Hydrogen between 298.15 and $1000 \mathrm{~K}$.

\begin{tabular}{|c|c|c|c|c|c|c|c|}
\hline$T / \mathrm{K}$ & $\begin{array}{l}\text { Exp. } C_{p} \\
{[24]}\end{array}$ & $\begin{array}{c}\text { Est. } C_{p} \\
{[18]}\end{array}$ & $\begin{array}{c}\mathrm{u}_{\mathrm{r}}\left(\mathrm{C}_{\mathrm{p}}\right) \\
\mathrm{x} 100\end{array}$ & $\begin{array}{l}\text { Est. } C_{p} \\
{[13 \mathrm{~b}]}\end{array}$ & $\begin{array}{c}\mathrm{u}_{\mathrm{r}}\left(\mathrm{C}_{\mathrm{p}}\right) \\
\mathrm{x} 100\end{array}$ & $\begin{array}{c}\text { Est. } C_{p} \\
{[20]}\end{array}$ & $\begin{array}{c}\mathrm{u}_{\mathrm{r}}\left(\mathrm{C}_{\mathrm{p}}\right) \\
\mathrm{x} 100\end{array}$ \\
\hline 298 & 28.84 & 28.88 & 0.13 & 28.77 & 0.24 & 28.87 & 0.10 \\
\hline 300 & 28.84 & 28.89 & 0.15 & 28.78 & 0.20 & 28.87 & 0.10 \\
\hline 400 & 29.18 & 29.13 & 0.19 & 29.17 & 0.05 & 28.93 & 0.87 \\
\hline 500 & 29.26 & 29.28 & 0.07 & 29.31 & 0.18 & 29.04 & 0.73 \\
\hline 600 & 29.32 & 29.38 & 0.20 & 29.36 & 0.11 & 29.21 & 0.38 \\
\hline 700 & 29.44 & 29.49 & 0.15 & 29.42 & 0.06 & 29.43 & 0.03 \\
\hline 800 & 29.63 & 29.64 & 0.03 & 29.58 & 0.16 & 29.69 & 0.23 \\
\hline 900 & 29.88 & 29.87 & 0.02 & 29.85 & 0.12 & 29.99 & 0.38 \\
\hline 1000 & 30.21 & 30.25 & 0.14 & 30.21 & 0.02 & 30.33 & 0.40 \\
\hline
\end{tabular}

It is obvious that polynomials described in Reid et al. [18] fit with smallest relative uncertainty to experimental data in the range 700 to $1000 \mathrm{~K}$. The heat capacity polynomial of isobutane according to Poling at al. [13b] exhibited excellent accuracy. However, it is a fifth order polynomial. Hence for consistency reason the polynomial after Reid et al. [18] is adopted for following estimations.

Figure 1, displays the excellent fit of adopted polynomials to experimental and data of gas phase heat capacities of reaction species.

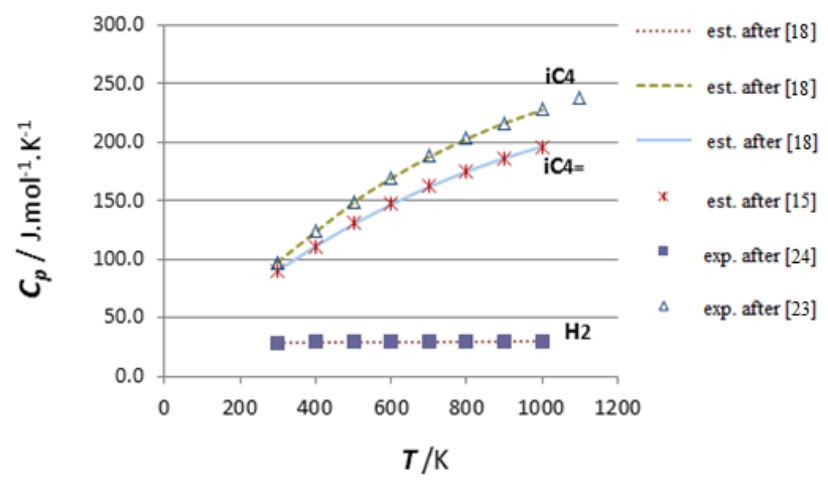

Figure 1. Estimated and experimentally measured gas phase heat capacity of isobutane $\left(\mathrm{iC}_{4}\right)$, isobutene $\left(\mathrm{iC}_{4}{ }^{ }\right)$, and hydrogen $\left(\mathrm{H}_{2}\right)$ as a function of temperature.

\section{4 $\Delta \boldsymbol{H}_{f, i}^{o}$ and $\Delta \boldsymbol{G}_{f, i}^{o}$ from Literature}

Many studies reported various values for $\Delta H_{f, i}^{o}, \Delta G_{f, i}^{o}$, and $\Delta S_{f, i}^{o}$ for isobutane and isobutene. Main sources and values are summarized in Table 7 and 8 . Selection of most reliable data follows lowest relative uncertainty; mainly for $\Delta H_{f, i}^{o}$ and $\Delta S_{f, i}^{o}$ as explained in section 2.1 .

Table 7. Reported Standard Enthalpy of Formation, Standard Gibbs Energy of Formation and Standard Entropy of Formation for Isobutane In Literature at $298.15 \mathrm{~K}$ and 1 bar.

\begin{tabular}{|c|c|c|c|c|c|c|c|}
\hline & & Prosen et al.[25] & Benson et al. [12] & Dom/Hea [7] & 97TRC ${ }^{\mathrm{a}}[19]$ & Scott [26] & Uncertainty ${ }^{b)}$ \\
\hline \multicolumn{8}{|c|}{$\Delta \boldsymbol{H}_{f}^{\boldsymbol{o}}(\mathbf{2 9 8 . 1 5}), \mathrm{kJ} \mathrm{mol}^{-1}$} \\
\hline & Experimental & $-135.65^{f}$ & -134.387 & $-134.18^{\mathrm{e}}$ & -134.99 & -134.387 & \multirow[t]{3}{*}{0.42} \\
\hline & Estimated & & -134.35 & -134.73 & & & \\
\hline & Relative Uncertainty ${ }^{c}$ ) & 0.97 & 0.03 & 0.12 & 0.48 & 0.03 & \\
\hline \multicolumn{8}{|c|}{$\Delta \boldsymbol{G}_{\boldsymbol{f}}^{\boldsymbol{o}}(\mathbf{2 9 8 . 1 5}), \mathrm{kJ} \cdot \mathrm{mol}^{-1}$} \\
\hline & Experimental & & $-21.23^{d}$ & -21.07 & $-21.47^{\mathrm{d}}$ & $-20.93^{\mathrm{d}}$ & \multirow[t]{3}{*}{0.16} \\
\hline & Estimated & & $-21.22^{d}$ & -20.78 & & & \\
\hline & Relative Uncertainty ${ }^{c)}$ & & 0.05 & 0.71 & 1.16 & 1.39 & \\
\hline \multicolumn{8}{|c|}{$\Delta S_{f}^{o}(298.15), \mathrm{J} \cdot \mathrm{mol}^{-1} \cdot \mathrm{K}^{-1}$} \\
\hline & Experimental & & $-379.53^{d}$ & -379.37 & $-380.85^{\mathrm{d}}$ & $-380.54^{\mathrm{d}}$ & \multirow[t]{3}{*}{0.43} \\
\hline & Estimated & & $-379.45^{d}$ & -382.07 & & & \\
\hline & Relative Uncertainty ${ }^{c)}$ & & 0.02 & 0.02 & 0.37 & 0.29 & \\
\hline a) & \multicolumn{7}{|c|}{ Poling et al. [13b]. Same figures reported in reference [19] } \\
\hline b) & \multirow{2}{*}{\multicolumn{7}{|c|}{$\begin{array}{l}\text { Standard uncertainty estimated after Eq.(27) under assumption rectangular probability } \\
\text { x } 100 \text { estimated based on Benson's el al. } 12]\end{array}$}} \\
\hline c) & & & & & & & \\
\hline d) & \multicolumn{7}{|c|}{$\begin{array}{l}\text { x100, estimated based on Benson's el al. [12] } \\
\text { corrected reference value from 1atm to } 1 \text { bar }\end{array}$} \\
\hline e) & \multicolumn{7}{|c|}{ quoted experimental value after [27], expanded uncertainty $\mp 0.63 \mathrm{~kJ}^{\mathrm{mol}}{ }^{-1}$, The reference used flame calorimeter } \\
\hline f) & \multicolumn{7}{|c|}{$\mp 0.54 \mathrm{~kJ} . \mathrm{mol}^{-1}$, given by source, The reference used Bomb calorimeter } \\
\hline
\end{tabular}


Table 8. Reported Standard Enthalpy of Formation, Standard Gibbs Energy of Formation and Standard Entropy of Formation for Isobutene. Standard Conditions: at $298.15 \mathrm{~K}$ and 1 bar.

\begin{tabular}{|c|c|c|c|c|c|c|c|}
\hline & $\begin{array}{c}\text { Prosen et } \\
\text { al.[25] }\end{array}$ & $\begin{array}{l}\text { Benson } \\
\text { et al.[12] }\end{array}$ & $\begin{array}{l}\text { Zwolinski } \\
\text { et al. [28] }\end{array}$ & $\begin{array}{c}\text { Dom/Hea } \\
{[7]}\end{array}$ & $\begin{array}{c}\text { 97TRC }^{\mathrm{a}} \\
{[19]}\end{array}$ & $\begin{array}{c}\text { Alb/Geh } \\
\text { [8] }\end{array}$ & Uncertainty $^{b)}$ \\
\hline \multicolumn{8}{|l|}{$\Delta \boldsymbol{H}_{f}^{o}(\mathbf{2 9 8 . 1 5}), \mathrm{kJ} \mathrm{mol}^{-1}$} \\
\hline Experimental & $-17.95^{f}$ & -16.89 & -16.9 & $-16.9^{e}$ & -17.1 & \multirow{3}{*}{-16.9} & \multirow[t]{3}{*}{0.31} \\
\hline Estimated & & $(-15.90)^{\mathrm{g}}$ & & -18.58 & & & \\
\hline Relative Uncertainty c) & 0.06 & 0.06 & 0.0 & 0.0 & 1.17 & & \\
\hline \multicolumn{8}{|l|}{$\Delta \boldsymbol{G}_{\boldsymbol{f}}^{\boldsymbol{o}}(\mathbf{2 9 8 . 1 5}), \mathrm{kJ}_{\mathrm{mol}} \mathrm{m}^{-1}$} \\
\hline Experimental & & $57.82^{d}$ & $58.05^{d}$ & 57.76 & $58.15^{\mathrm{d}}$ & \multirow{3}{*}{58.17} & \multirow[t]{3}{*}{0.11} \\
\hline Estimated & & $58.56^{d}$ & & 55.57 & & & \\
\hline Relative Uncertainty ${ }^{c)}$ & & 0.60 & 0.21 & 0.70 & 0.03 & & \\
\hline \multicolumn{8}{|l|}{$\Delta S_{f}^{o}(\mathbf{2 9 8 . 1 5}), \mathrm{J} \cdot \mathrm{mol}^{-1} \cdot \mathrm{K}^{-1}$} \\
\hline Experimental & & $-250.58^{d}$ & & $-250.41^{d}$ & $-252.39^{d}$ & $251.38^{\mathrm{d}, \mathrm{h}}$ & \multirow[t]{3}{*}{0.57} \\
\hline Estimated & & $-249.74^{d}$ & & $-248.60^{d}$ & & $-250.19^{d}$ & \\
\hline Relative Uncertainty ${ }^{c)}$ & & 0.15 & & 0.09 & 0.88 & 0.48 & \\
\hline
\end{tabular}

a), b) and d) see Table 7 foot note

c) $\mathrm{x} 100$, estimated based on reference [8]

e) quoted experimental value, reference not given

f) $\mp 1.09 \mathrm{~kJ} \mathrm{~mol}^{-1}$, given by the source g) Benson [17]

h) Scott [26]

Table 9. Standard Molar Enthalpy and Standard Molar Gibbs Free Energy of Isobutane Dehydrogenation Reaction Based on Formation Energies Given by Several Authors, in kJ.mol ${ }^{-1}$.

\begin{tabular}{|c|c|c|c|c|c|c|c|}
\hline & $\begin{array}{l}\text { Prosen } \\
\text { et al.[25] }\end{array}$ & $\begin{array}{c}\text { Benson } \\
\text { et al.[12] }\end{array}$ & $\begin{array}{c}97 \mathrm{TRC}^{\mathrm{a}} \\
{[19]}\end{array}$ & $\begin{array}{c}\text { Dom/Hea } \\
{[7]}\end{array}$ & This work & $\max . \Delta^{\mathrm{b}}$ & $\begin{array}{c}\text { Expanded } \\
\text { Uncertainty }\end{array}$ \\
\hline$\Delta_{R} H^{o}(298.15)$ & $117.70^{c}$ & 117.50 & 117.89 & 117.28 & 117.49 & 0.61 & 1.04 \\
\hline$\Delta_{R} G^{o}(298.15)$ & & 79.05 & 79.62 & 78.83 & 78.99 & 0.79 & 0.38 \\
\hline
\end{tabular}

a) Poling et al. [13b]

b) maximum difference between highest and lowest values

c) $\mp 1.63 \mathrm{~kJ} . \mathrm{mol}^{-1}$

Due to diversity of measurement/estimation techniques, it appears convenient to consider that all thermodynamic figures given in these tables have equal probability to be correct. Uncertainty is then estimated following to the method described in [16] for rectangular distribution of type B. Standard uncertainty is therefore calculated from the following equation:

$u(x)=a / \sqrt{3}$

where $x$ is the variable; for $\Delta H_{f, i}^{o}$ or $\Delta G_{f, i}^{o}, a$ is the half of the interval between measurand's upper- and lower bounds.

The values for each compound listed in bold were selected for all subsequent calculations. Maximum difference in reported $\Delta H_{f, i}^{o}$ values according to Tables 7 and 8 is 1.47 $\mathrm{kJ} . \mathrm{mol}^{-1}$. Whereas maximum difference in reported values $\Delta G_{f, i}^{o}$ is $0.54 \mathrm{~kJ} \cdot \mathrm{mol}^{-1}$. These differences are generally accounted as usual fluctuation in reported thermodynamic data.

\section{Results and Discussion}

\subsection{Sensitivity Analysis}

Equilibrium constant, $K_{T}$, is very sensitive to the thermodynamic quantity, $\Delta_{R} G_{T}^{o}$. In following it is demonstrated how much available information can affect the value of $K_{T}$ and other thermodynamic parameters for isobutane dehydrogenation reaction.

Table 9 compares calculated standard molar reaction enthalpy and standard molar Gibbs free energy, while Table 10 compares the equilibrium constants. Maximum difference in $\Delta_{\mathrm{R}} H_{298.15}^{o}$ and in $\Delta_{\mathrm{R}} G_{298.15}^{o}$ values is 0.61 and $0.79 \mathrm{~kJ} . m o l^{-}$ 1 , respectively. Standard molar enthalpy of isobutane dehydrogenation reaction is $118 \pm 1.0 \mathrm{~kJ} \mathrm{~mol}^{-1}$, while standard molar Gibbs free energy equals to $79.0 \pm 0.4 \mathrm{~kJ}^{\mathrm{m}} \mathrm{mol}^{-}$ 1 .

It is to recognize that mathematical analysis of uncertainties in thermodynamic parameters usually follows reasonable assumptions. Expanded uncertainty of standard molar enthalpy, $1.0 \mathrm{~kJ}^{\mathrm{mol}}{ }^{-1}$, for example, is calculated based on equal probability assumption for all literary available enthalpies of formations since they have different origin and methods as described above.

The calculation of uncertainty in equilibrium constant is rather more complicated. Thus reasonable assumption must be made to simplify the calculation. One could make here two different, however both seem reasonable, assumptions: a. Each of temperature and heat capacity coefficients have negligible error:

In this case expanded uncertainty of equilibrium constant, $U_{e}\left(K_{T}\right)$, could be calculated according to Eqs. (4), (10), and (21) - (24):

$U_{e}=k u_{c}\left(x_{i}\right)$

whereby $k=2$ is the coverage factor for $95 \%$ confidence level, $u_{\mathrm{c}}\left(x_{i}\right)$ is the combined uncertainty (propagated uncertainty), calculated according to Eq. (22):

$u_{c}^{2}(y)=\sum_{i=1}^{N}\left(\frac{\partial f}{\partial x_{i}}\right)^{2} u^{2}\left(x_{i}\right)$

where $f$ is the function according to Eq. (1) or (3), while $u\left(x_{i}\right)$ values are calculated based on Eq. (20) and related values from Tables 7 and 8 .

$u_{c}^{2}\left(\ln K_{T}\right)=\frac{u_{c}^{2}\left(\Delta_{R} H_{298.15}^{o}\right)}{(R T)^{2}}+\frac{u_{c}^{2}\left(\Delta_{R} S_{298.15}^{o}\right)}{R^{2}}$ 


$$
u_{c}\left(K_{T}\right)=K_{T} \cdot u_{c}\left(\ln K_{T}\right)
$$

whereby (according to Eq. 22 and Tables 7-9):

$u_{c}\left(\Delta_{R} H_{298.15}^{o}\right)=0.52 \mathrm{~kJ}^{\circ} \mathrm{mol}^{-1}$

$u_{c}\left(\Delta_{R} S_{298.15}^{o}\right)=0.7 \mathrm{~J} \cdot \mathrm{mol}^{-1} \cdot \mathrm{K}^{-1}$

b. Temperature only has negligible error:

In this case uncertainty is propagated according to Eqs. (4) through (10) as follows: From Eq.(5) and Eq.(22):

$u_{c}^{2}\left(\Delta_{R} H_{T}^{o}\right)=u_{c}^{2}\left(\Delta_{R} H_{298.15}^{o}\right)+(T-298.15)^{2} \cdot u_{c}^{2}\left(\Delta_{R} C_{p}\right)$

whereby: $u_{c}\left(\Delta_{R} C_{p}\right)$ is the combined uncertainty in heat capacity of reaction mixture at temperature $T$ according to Eqs. (7), (22) and (28). Uncertainty in heat capacity for each reaction species $u\left(\mathrm{C}_{p i}\right)$ is estimated using differences $\Delta C p_{i}$ between experimental- and $C_{p i}$-data after Reid et al. [18]. The variance of differences for concerned $T$ range between 700 and $1000 \mathrm{~K}(N=4$ points, typed in bold in the tables $4-6)$ are calculated using Eqs. (26) and (27), following to the method described in [16] for "type A evaluation" under assumption that individual observations of $C_{p i}$ differ in values due to random effects.

The further uncertainty calculation is accomplished using Eqs. (27 through 31):

$s\left(C_{p_{i}}\right)=\sqrt{\frac{1}{N-1} \sum_{1}^{N=4} \Delta C p_{i}}$

$u_{c}^{2}\left(\mathrm{Cp}_{i}\right)=s^{2}\left(C_{p_{i}}\right) / N$

Intermediate results: $u_{c}^{2}\left(\mathrm{Cp}_{i C 4}\right)=0.172, u_{c}^{2}\left(\mathrm{Cp}_{i C 4}=\right)=0.04$, $u_{c}^{2}\left(\mathrm{Cp}_{H 2}\right)=0.0001\left(\mathrm{~J} \cdot \mathrm{mol}^{-1} \cdot \mathrm{K}^{-1}\right)^{2}$

From Eq.(7) and Eq.(22):

$u_{c}^{2}\left(\Delta_{\mathrm{R}} C_{p}\right)=\sum u_{c}^{2}\left(C_{p_{i}}\right)$

Eq.(25) can be resolved after T, using following input data: $u_{c}\left(\Delta_{R} H_{298.15}^{o}\right)=0.52 \mathrm{~kJ} . \mathrm{mol}^{-1}$ $u_{c}\left(\Delta_{\mathrm{R}} C_{p}\right)=0.46 \mathrm{~J} \cdot \mathrm{mol}^{-1} \cdot \mathrm{K}^{-1}$

From Eq.(6) and Eq.(22):

$u_{c}^{2}\left(\Delta_{\mathrm{R}} S_{T}^{o}\right)=u_{c}^{2}\left(\Delta_{\mathrm{R}} S_{298.15}^{o}\right)+\left(\ln \frac{T}{298.15}\right)^{2} \cdot u_{c}^{2}\left(\Delta_{\mathrm{R}} C_{p}\right)$

Eq.(29) can be resolved after $T$, using following input data: $u_{c}\left(\Delta_{R} S_{298.15}^{o}\right)=0.7 \mathrm{~J} \cdot \mathrm{mol}^{-1} \cdot \mathrm{K}^{-1}$ $u_{c}\left(\Delta_{\mathrm{R}} C_{p}\right)=0.46 \mathrm{~J} \cdot \mathrm{mol}^{-1} \cdot \mathrm{K}^{-1}$

From Eq.(11) and Eq.(22):

$u_{c}^{2}\left(\Delta_{\mathrm{R}} G_{T}^{o}\right)=u_{c}^{2}\left(\Delta_{\mathrm{R}} H_{T}^{o}\right)+T^{2} \cdot u_{c}^{2}\left(\Delta_{\mathrm{R}} S_{T}^{o}\right)$

Eq. (30) to be resolved after $T$ and outcomes of Eqs.(29) and (25) From equations (4), (22) and (24):

$u_{c}\left(K_{T}\right)=K_{T} \cdot \frac{u_{c}\left(\Delta_{\mathrm{R}} G_{T}^{o}\right)}{R T}$

Expanded uncertainty $U_{e}\left(K_{T}\right)$ is then calculated according to Eq. (21).

Table 10 reports equilibrium constant with expanded uncertainties ranging between 21.5 and $23.5 \%$ of $K$ value following to the first assumption, versus 26 to $26.5 \%$ following to the second assumption. It is apparent that both assumptions give reasonably matching uncertainty. Besides, one can see that slight difference in standard molar reaction enthalpy $\left(0.61 \mathrm{~kJ}^{\mathrm{mol}}{ }^{-1}\right.$, Table 9$)$ or in standard molar Gibbs

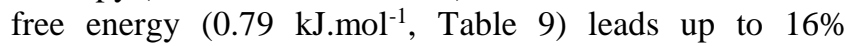
difference in equilibrium constant at high temperatures. Thus it is important to select accurate input data from literature for calculation. Figure 2 shows equilibrium constants calculated according to various data sources, under usage of same $C_{p^{-}}$ Temperature regression function. All values are within the given uncertainty limits, using first assumption.

Table 10. Equilibrium Constant $K_{T}$ of Isobutane Dehydrogenation Reaction at Various Temperatures, Based on Several Data Sources.

\begin{tabular}{ccccccc}
\hline$T / \mathrm{K}$ & {$[7]$} & {$[13 \mathrm{~b}]$} & {$[12]$} & This work & $U_{e}\left(\mathrm{~K}_{\mathrm{T}}\right)^{\mathrm{a}}$ & $U_{e}\left(\mathrm{~K}_{\mathrm{T}}\right)^{\mathrm{b}}$ \\
\hline 773.15 & 0.12 & 0.10 & 0.11 & $\mathbf{0 . 1 2}$ & $\mathbf{0 . 0 3}$ & $\mathbf{0 . 0 3}$ \\
783.15 & 0.15 & 0.13 & 0.14 & $\mathbf{0 . 1 5}$ & $\mathbf{0 . 0 4}$ & $\mathbf{0 . 0 3}$ \\
793.15 & 0.19 & 0.16 & 0.18 & $\mathbf{0 . 1 9}$ & $\mathbf{0 . 0 5}$ & $\mathbf{0 . 0 4}$ \\
803.15 & 0.24 & 0.20 & 0.23 & $\mathbf{0 . 2 4}$ & $\mathbf{0 . 0 6}$ & $\mathbf{0 . 0 5}$ \\
813.15 & 0.30 & 0.25 & 0.29 & $\mathbf{0 . 3 0}$ & $\mathbf{0 . 0 8}$ & $\mathbf{0 . 0 7}$ \\
823.15 & 0.37 & 0.32 & 0.36 & $\mathbf{0 . 3 7}$ & $\mathbf{0 . 1 0}$ & $\mathbf{0 . 0 8}$ \\
833.15 & 0.46 & 0.39 & 0.45 & $\mathbf{0 . 4 6}$ & $\mathbf{0 . 1 2}$ & $\mathbf{0 . 1 0}$ \\
843.15 & 0.57 & 0.49 & 0.55 & $\mathbf{0 . 5 6}$ & $\mathbf{0 . 1 5}$ & $\mathbf{0 . 1 3}$ \\
853.15 & 0.70 & 0.60 & 0.68 & $\mathbf{0 . 6 9}$ & $\mathbf{0 . 1 8}$ & $\mathbf{0 . 1 5}$ \\
863.15 & 0.86 & 0.73 & 0.83 & $\mathbf{0 . 8 5}$ & $\mathbf{0 . 2 2}$ & $\mathbf{0 . 1 9}$ \\
873.15 & 1.04 & 0.89 & 1.01 & $\mathbf{1 . 0 3}$ & $\mathbf{0 . 2 7}$ & $\mathbf{0 . 2 3}$ \\
883.15 & 1.26 & 1.08 & 1.22 & $\mathbf{1 . 2 5}$ & $\mathbf{0 . 3 3}$ & $\mathbf{0 . 2 7}$ \\
893.15 & 1.52 & 1.30 & 1.47 & $\mathbf{1 . 5 0}$ & $\mathbf{0 . 3 9}$ & $\mathbf{0 . 3 3}$ \\
903.15 & 1.82 & 1.56 & 1.76 & $\mathbf{1 . 8 0}$ & $\mathbf{0 . 4 7}$ & $\mathbf{0 . 3 9}$ \\
913.15 & 2.17 & 1.87 & 2.11 & $\mathbf{2 . 1 6}$ & $\mathbf{0 . 5 6}$ & $\mathbf{0 . 4 7}$ \\
923.15 & 2.59 & 2.22 & 2.51 & $\mathbf{2 . 5 7}$ & $\mathbf{0 . 6 7}$ & $\mathbf{0 . 5 6}$ \\
\hline \multicolumn{5}{c}{ Expanded uncertainty, assuming that both of temperature and heat } \\
capacity coefficients have negligible error. \\
b) Expanded uncertainty, assuming that the temperature only has
\end{tabular}

b) Expanded uncertainty, assuming that the temperature only has negligible error.

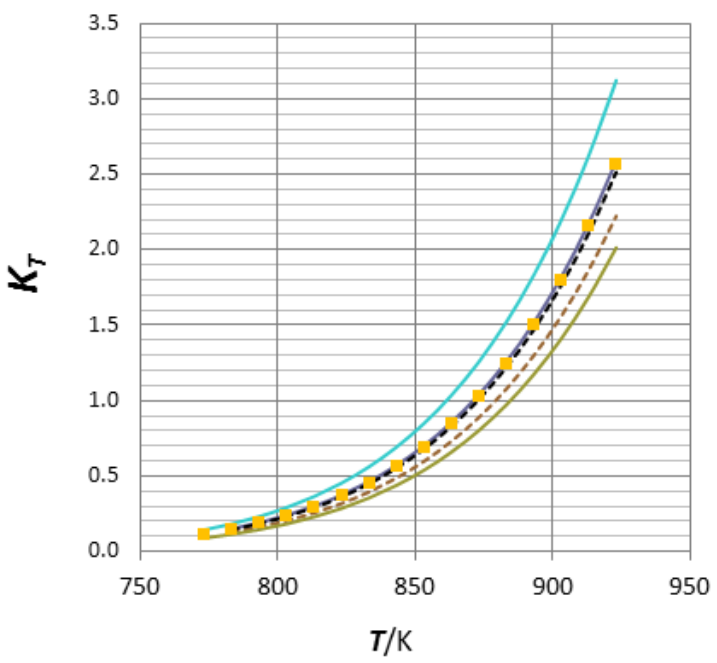

Figure 2: Equilibrium constant variation with temperature for isobutane dehydrogenation reaction using several input data

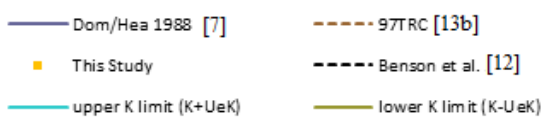

3.2 Equilibrium of Isobutane Dehydrogenation Reaction

The set of data selected above has the advantage of including "best available" thermodynamic quantities: $\Delta H_{f, i}^{o}$, and $\Delta G_{f, i}^{o}$, and $C_{p_{i}}^{o}$ and best fitting $C_{p}-T$ regression function in literature. The reaction enthalpy, the Gibbs free energy change and equilibrium constant of isobutane dehydrogenation reaction have been calculated for industrially relevant temperatures. Results are shown in Tables 10 and 11 .

The reaction is strongly endothermic. Reaction enthalpy, $\Delta_{\mathrm{R}} H_{T}^{o}$, ranges between $122 \pm 1.1$ and $123 \pm 1.2$ $\mathrm{kJ} . \mathrm{mol}^{-1}$. Free Gibbs energy, $\Delta_{\mathrm{R}} G_{T}^{o}$, shows that isobutane dehydrogenation becomes thermodynamically favorable at temperatures higher than $873.15 \mathrm{~K}$. The values of equilibrium constant $K_{T}$ varies with temperature between $0.12 \pm 0.03$ and $2.6 \pm 0.7$. 
Table 11. Calculated Reaction Enthalpy and Free Gibbs Energy at Various Temperatures for Isobutane Dehydrogenation, in $\mathrm{kJmol}^{-1}$.

\begin{tabular}{cccccc}
\hline$T^{\circ} \mathrm{C}$ & $T \mathrm{~K}$ & $\Delta_{\mathrm{R}} H_{T}^{o}$ & $U_{e}\left(\Delta_{\mathrm{R}} H_{T}^{o}\right)^{\mathrm{a}}$ & $\Delta_{\mathrm{R}} G_{T}^{o}$ & $U_{e}\left(\Delta_{\mathrm{R}} G_{T}^{o}\right)^{\mathrm{a}}$ \\
\hline 500 & 773.15 & 122.38 & 1.13 & 13.84 & 1.70 \\
510 & 783.15 & 122.40 & 1.13 & 12.43 & 1.72 \\
520 & 793.15 & 122.42 & 1.14 & 11.03 & 1.74 \\
530 & 803.15 & 122.44 & 1.14 & 9.63 & 1.76 \\
540 & 813.15 & 122.45 & 1.14 & 8.22 & 1.78 \\
550 & 823.15 & 122.47 & 1.15 & 6.82 & 1.80 \\
560 & 833.15 & 122.48 & 1.15 & 5.41 & 1.82 \\
570 & 843.15 & 122.49 & 1.15 & 4.01 & 1.84 \\
580 & 853.15 & 122.50 & 1.16 & 2.60 & 1.86 \\
590 & 863.15 & 122.51 & 1.16 & 1.19 & 1.88 \\
600 & 873.15 & 122.51 & 1.17 & -0.21 & 1.90 \\
610 & 883.15 & 122.52 & 1.17 & -1.62 & 1.92 \\
620 & 893.15 & 122.52 & 1.18 & -3.02 & 1.94 \\
630 & 903.15 & 122.52 & 1.18 & -4.43 & 1.96 \\
640 & 913.15 & 122.52 & 1.18 & -5.83 & 1.98 \\
650 & 923.15 & 122.52 & 1.19 & -7.24 & 2.00 \\
\hline Expandy
\end{tabular}

a) Expanded uncertainty calculated under assumption that only temperature measurements has negligible error.

From industrial point of view, to achieve reasonable equilibrium conversion, the reaction is usually operated at high temperature and low pressure in Catofin process. FBD4 and Oleflex processes, however, operate at pressure slightly above atmospheric. Therefore, equilibrium conversion has been calculated for wide range of temperatures and pressures. Calculations followed Eq. (17). Results are presented in Tables 12 through 16 and Figure 3.

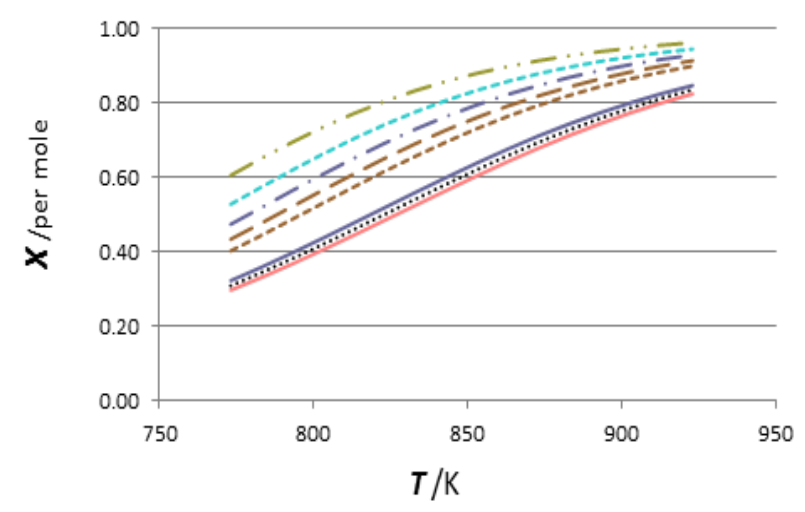

Figure 3: Isobutane conversion as a function of temperature in isobutane dehydrogenation reaction carried out under several presures.

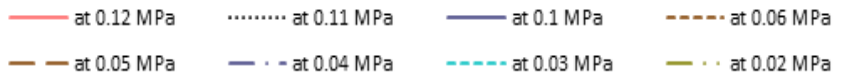

Table 12. Equilibrium Conversion per Mole Isobutane at Various Temperatures and Pressures, Feed Gas without Diluent. Expanded Uncertainties are Given in Table 13.

\begin{tabular}{ccccccccccc}
\hline$T / \mathrm{C}$ & $T / \mathrm{K}$ & $0.12 \mathrm{MPa}$ & $0.11 \mathrm{MPa}$ & $0.1 \mathrm{MPa}$ & $0.07 \mathrm{MPa}$ & $0.06 \mathrm{MPa}$ & $0.05 \mathrm{MPa}$ & $0.04 \mathrm{MPa}$ & $0.03 \mathrm{MPa}$ & $0.02 \mathrm{MPa}$ \\
\hline 500 & 773.15 & 0.2971 & 0.3091 & 0.3227 & 0.3773 & 0.4028 & 0.4343 & 0.4745 & 0.5284 & 0.6062 \\
510 & 783.15 & 0.3314 & 0.3445 & 0.3592 & 0.4179 & 0.4449 & 0.4780 & 0.5198 & 0.5749 & 0.6523 \\
520 & 793.15 & 0.3678 & 0.3818 & 0.3975 & 0.4598 & 0.4881 & 0.5224 & 0.5651 & 0.6204 & 0.6958 \\
530 & 803.15 & 0.4058 & 0.4207 & 0.4374 & 0.5026 & 0.5318 & 0.5668 & 0.6096 & 0.6640 & 0.7361 \\
540 & 813.15 & 0.4451 & 0.4607 & 0.4782 & 0.5454 & 0.5750 & 0.6101 & 0.6524 & 0.7050 & 0.7727 \\
550 & 823.15 & 0.4852 & 0.5014 & 0.5194 & 0.5877 & 0.6173 & 0.6518 & 0.6929 & 0.7429 & 0.8055 \\
560 & 833.15 & 0.5255 & 0.5421 & 0.5604 & 0.6289 & 0.6579 & 0.6914 & 0.7306 & 0.7773 & 0.8343 \\
570 & 843.15 & 0.5657 & 0.5824 & 0.6007 & 0.6682 & 0.6963 & 0.7283 & 0.7651 & 0.8081 & 0.8593 \\
580 & 853.15 & 0.6051 & 0.6217 & 0.6398 & 0.7054 & 0.7321 & 0.7622 & 0.7963 & 0.8354 & 0.8809 \\
590 & 863.15 & 0.6432 & 0.6595 & 0.6771 & 0.7399 & 0.7650 & 0.7929 & 0.8241 & 0.8593 & 0.8994 \\
600 & 873.15 & 0.6795 & 0.6953 & 0.7122 & 0.7715 & 0.7948 & 0.8204 & 0.8486 & 0.8800 & 0.9151 \\
610 & 883.15 & 0.7138 & 0.7288 & 0.7449 & 0.8002 & 0.8216 & 0.8448 & 0.8701 & 0.8978 \\
620 & 893.15 & 0.7456 & 0.7598 & 0.7748 & 0.8259 & 0.8453 & 0.8662 & 0.8887 & 0.9130 & 0.9393 \\
630 & 903.15 & 0.7749 & 0.7881 & 0.8020 & 0.8487 & 0.8662 & 0.8848 & 0.9047 & 0.9259 & 0.9488 \\
640 & 913.15 & 0.8015 & 0.8137 & 0.8265 & 0.8689 & 0.8845 & 0.9010 & 0.9184 & 0.9369 & 0.9566 \\
650 & 923.15 & 0.8256 & 0.8367 & 0.8484 & 0.8865 & 0.9003 & 0.9149 & 0.9302 & 0.9463 & 0.9632 \\
\hline
\end{tabular}

Table 13. Expanded Uncertainty $U_{e}(X)$ in Equilibrium Conversion of Table 12, per Mole Isobutane at Tand $P{ }^{\mathrm{A}}$

\begin{tabular}{ccccccccccc}
\hline$T / \mathrm{C}$ & $T / \mathrm{K}$ & $0.12 \mathrm{MPa}$ & $0.11 \mathrm{MPa}$ & $0.1 \mathrm{MPa}$ & $0.07 \mathrm{MPa}$ & $0.06 \mathrm{MPa}$ & $0.05 \mathrm{MPa}$ & $0.04 \mathrm{MPa}$ & $0.03 \mathrm{MPa}$ & $0.02 \mathrm{MPa}$ \\
\hline 500 & 773.15 & 0.0348 & 0.0363 & 0.0379 & 0.0445 & 0.0476 & 0.0516 & 0.0568 & 0.0641 & 0.0754 \\
510 & 783.15 & 0.0387 & 0.0403 & 0.0420 & 0.0492 & 0.0526 & 0.0569 & 0.0625 & 0.0703 & 0.0823 \\
520 & 793.15 & 0.0428 & 0.0445 & 0.0464 & 0.0542 & 0.0579 & 0.0625 & 0.0684 & 0.0767 & 0.0892 \\
530 & 803.15 & 0.0472 & 0.0490 & 0.0511 & 0.0595 & 0.0634 & 0.0683 & 0.0746 & 0.0832 & 0.0960 \\
540 & 813.15 & 0.0517 & 0.0537 & 0.0559 & 0.0649 & 0.0691 & 0.0742 & 0.0809 & 0.0898 & 0.1026 \\
550 & 823.15 & 0.0565 & 0.0586 & 0.0610 & 0.0705 & 0.0749 & 0.0803 & 0.0872 & 0.0962 & 0.1089 \\
560 & 833.15 & 0.0615 & 0.0637 & 0.0663 & 0.0763 & 0.0809 & 0.0864 & 0.0934 & 0.1024 & 0.1147 \\
570 & 843.15 & 0.0666 & 0.0690 & 0.0717 & 0.0821 & 0.0868 & 0.0925 & 0.0995 & 0.1083 & 0.1199 \\
580 & 853.15 & 0.0719 & 0.0744 & 0.0772 & 0.0880 & 0.0927 & 0.0984 & 0.1053 & 0.1137 & 0.1245 \\
590 & 863.15 & 0.0773 & 0.0799 & 0.0827 & 0.0937 & 0.0985 & 0.1040 & 0.1107 & 0.1187 & 0.1285 \\
600 & 873.15 & 0.0827 & 0.0854 & 0.0883 & 0.0993 & 0.1039 & 0.1093 & 0.1156 & 0.1230 \\
610 & 883.15 & 0.0881 & 0.0908 & 0.0937 & 0.1045 & 0.1091 & 0.1142 & 0.1201 & 0.1269 & 0.1319 \\
620 & 893.15 & 0.0934 & 0.0961 & 0.0990 & 0.1095 & 0.1138 & 0.1186 & 0.1240 & 0.1302 & 0.1372 \\
630 & 903.15 & 0.0985 & 0.1011 & 0.1040 & 0.1140 & 0.1181 & 0.1225 & 0.1274 & 0.1330 \\
640 & 913.15 & 0.1034 & 0.1059 & 0.1086 & 0.1182 & 0.1219 & 0.1260 & 0.1304 & 0.1353 \\
650 & 923.15 & 0.1080 & 0.1104 & 0.1130 & 0.1218 & 0.1253 & 0.1289 & 0.1329 & 0.1373 & 0.1407 \\
\hline
\end{tabular}

a) Calculated based on equations (21) and (22), whereby $\mathrm{f}$ is the function according to equation (19)

$$
\text { The resulted equation is the following: }\left(\frac{u_{c}(X)}{X}\right)^{2}=\left(\frac{1}{2}\right)^{2}\left(\frac{u_{c}\left(K_{T}\right)}{K_{T}}\right)^{2}+\left(\frac{1}{2}\right)^{2}\left(\frac{u_{c}\left(K_{T}\right)}{P+K_{T}}\right)^{2}
$$


Table 14. Equilibrium Conversion per Mole Isobutane at Technically Relevant Temperatures and Pressures When 1 Volume Hydrogen is Mixed to 100 Volumes Isobutane Feed.

\begin{tabular}{ccccccc}
\hline \multicolumn{4}{c}{$0.12 \mathrm{MPa}$} & \multicolumn{2}{c}{$0.11 \mathrm{MPa}$} & \multicolumn{2}{c}{$0.1 \mathrm{MPa}$} \\
\hline$T / \mathrm{K}$ & $X$ & $U_{e}{ }^{a)}$ & $X$ & $U_{e}{ }^{a)}$ & $X$ & $U_{e}{ }^{a)}$ \\
\hline 773.15 & 0.2937 & 0.0391 & 0.3057 & 0.0407 & 0.3193 & 0.0425 \\
783.15 & 0.3281 & 0.0437 & 0.3412 & 0.0454 & 0.3560 & 0.0474 \\
793.15 & 0.3646 & 0.0486 & 0.3787 & 0.0505 & 0.3946 & 0.0526 \\
803.15 & 0.4029 & 0.0538 & 0.4179 & 0.0558 & 0.4346 & 0.0581 \\
813.15 & 0.4423 & 0.0593 & 0.4580 & 0.0614 & 0.4756 & 0.0638 \\
823.15 & 0.4826 & 0.0651 & 0.4989 & 0.0673 & 0.5170 & 0.0698 \\
833.15 & 0.5232 & 0.0712 & 0.5399 & 0.0735 & 0.5582 & 0.0760 \\
843.15 & 0.5635 & 0.0775 & 0.5804 & 0.0799 & 0.5988 & 0.0824 \\
853.15 & 0.6031 & 0.0841 & 0.6198 & 0.0864 & 0.6380 & 0.0890 \\
863.15 & 0.6414 & 0.0908 & 0.6578 & 0.0931 & 0.6755 & 0.0956 \\
873.15 & 0.6779 & 0.0976 & 0.6938 & 0.0999 & 0.7108 & 0.1023 \\
883.15 & 0.7123 & 0.1044 & 0.7275 & 0.1066 & 0.7436 & 0.1090 \\
893.15 & 0.7443 & 0.1112 & 0.7586 & 0.1133 & 0.7737 & 0.1155 \\
903.15 & 0.7738 & 0.1177 & 0.7871 & 0.1198 & 0.8011 & 0.1219 \\
913.15 & 0.8005 & 0.1241 & 0.8128 & 0.1260 & 0.8257 & 0.1280 \\
923.15 & 0.8247 & 0.1301 & 0.8359 & 0.1319 & 0.8476 & 0.1337 \\
\hline Calculated based on equations (21) and (32), whereby h assumed negligible
\end{tabular}

Table 15. Equilibrium Conversion per Mole Isobutane at Technically Relevant Temperatures and Pressures When 5 Volume Hydrogen is Mixed to 100 Volumes Isobutane Feed.

\begin{tabular}{ccccccc}
\hline & \multicolumn{2}{c}{$0.12 \mathrm{MPa}$} & \multicolumn{2}{c}{$0.11 \mathrm{MPa}$} & \multicolumn{2}{c}{$0.1 \mathrm{MPa}$} \\
\cline { 2 - 7 }$T / \mathrm{K}$ & $X$ & $U_{e}{ }^{a)}$ & $X$ & $U_{e}{ }^{a)}$ & $X$ & $U_{e}{ }^{a)}$ \\
\hline 773.15 & 0.2805 & 0.0373 & 0.2927 & 0.0390 & 0.3066 & 0.0409 \\
783.15 & 0.3156 & 0.0420 & 0.3289 & 0.0438 & 0.3439 & 0.0459 \\
793.15 & 0.3527 & 0.0470 & 0.3670 & 0.0490 & 0.3831 & 0.0512 \\
803.15 & 0.3916 & 0.0523 & 0.4068 & 0.0545 & 0.4239 & 0.0569 \\
813.15 & 0.4317 & 0.0579 & 0.4478 & 0.0602 & 0.4656 & 0.0628 \\
823.15 & 0.4728 & 0.0638 & 0.4894 & 0.0663 & 0.5078 & 0.0691 \\
833.15 & 0.5141 & 0.0700 & 0.5311 & 0.0726 & 0.5498 & 0.0756 \\
843.15 & 0.5552 & 0.0764 & 0.5723 & 0.0792 & 0.5911 & 0.0824 \\
853.15 & 0.5955 & 0.0830 & 0.6126 & 0.0860 & 0.6311 & 0.0893 \\
863.15 & 0.6345 & 0.0898 & 0.6512 & 0.0929 & 0.6693 & 0.0963 \\
873.15 & 0.6717 & 0.0967 & 0.6879 & 0.0999 & 0.7052 & 0.1034 \\
883.15 & 0.7068 & 0.1036 & 0.7222 & 0.1068 & 0.7387 & 0.1103 \\
893.15 & 0.7394 & 0.1104 & 0.7540 & 0.1136 & 0.7694 & 0.1171 \\
903.15 & 0.7694 & 0.1171 & 0.7830 & 0.1202 & 0.7972 & 0.1237 \\
913.15 & 0.7967 & 0.1235 & 0.8092 & 0.1266 & 0.8223 & 0.1299 \\
923.15 & 0.8213 & 0.1296 & 0.8328 & 0.1325 & 0.8447 & 0.1357 \\
\hline a) see Table 14 foot note & & & &
\end{tabular}

Table 17. Molar Fractions of Reaction Species in Equilibrium at Different Temperatures and Pressures, Isobutane Feed without Diluent. Expanded Uncertainties are Given in Table 18.

\begin{tabular}{|c|c|c|c|c|c|c|c|c|c|c|c|c|c|c|}
\hline \multirow[b]{2}{*}{$T / \mathrm{K}$} & \multicolumn{3}{|c|}{$0.12 \mathrm{MPa}$} & \multicolumn{3}{|c|}{$0.11 \mathrm{MPa}$} & \multicolumn{3}{|c|}{$0.1 \mathrm{MPa}$} & \multicolumn{3}{|c|}{$0.07 \mathrm{MPa}$} & \multicolumn{2}{|c|}{$0.06 \mathrm{MPa}$} \\
\hline & $\boldsymbol{y}_{C 4 H 10}$ & $\boldsymbol{y}_{\mathrm{C} 4 \mathrm{H} 8}$ & $\boldsymbol{y}_{H 2}$ & $\boldsymbol{y}_{C 4 H 10}$ & $\boldsymbol{y}_{C 4 H 8}$ & $\boldsymbol{y}_{H 2}$ & $y_{C 4 H 10}$ & $\boldsymbol{y}_{C 4 H 8}$ & $\boldsymbol{y}_{H 2}$ & $\boldsymbol{y}_{C 4 H 10}$ & $\boldsymbol{y}_{C 4 H 8}$ & $\boldsymbol{y}_{\mathrm{H} 2}$ & $\boldsymbol{y}_{C 4 H 10}$ & $\boldsymbol{y}_{C 4 H 8}$ \\
\hline 773.15 & 0.5419 & 0.2291 & 0.2291 & 0.5278 & 0.2361 & 0.2361 & 0.5121 & 0.2439 & 0.2439 & 0.4521 & 0.2739 & 0.2739 & 0.4257 & 0.2871 \\
\hline 783.15 & 0.5021 & 0.2489 & 0.2489 & 0.4876 & 0.2562 & 0.2562 & 0.4715 & 0.2643 & 0.2643 & 0.4106 & 0.2947 & 0.2947 & 0.3841 & 0.3079 \\
\hline 793.15 & 0.4622 & 0.2689 & 0.2689 & 0.4474 & 0.2763 & 0.2763 & 0.4311 & 0.2845 & 0.2845 & 0.3700 & 0.3150 & 0.3150 & 0.3440 & 0.3280 \\
\hline 803.15 & 0.4227 & 0.2887 & 0.2887 & 0.4077 & 0.2961 & 0.2961 & 0.3914 & 0.3043 & 0.3043 & 0.3310 & 0.3345 & 0.3345 & 0.3057 & 0.3472 \\
\hline 813.15 & 0.3840 & 0.3080 & 0.3080 & 0.3692 & 0.3154 & 0.3154 & 0.3530 & 0.3235 & 0.3235 & 0.2942 & 0.3529 & 0.3529 & 0.2698 & 0.3651 \\
\hline 823.15 & 0.3467 & 0.3267 & 0.3267 & 0.3321 & 0.3340 & 0.3340 & 0.3163 & 0.3418 & 0.3418 & 0.2597 & 0.3702 & 0.3702 & 0.2366 & 0.3817 \\
\hline 833.15 & 0.3110 & 0.3445 & 0.3445 & 0.2969 & 0.3516 & 0.3516 & 0.2817 & 0.3592 & 0.3592 & 0.2279 & 0.3861 & 0.3861 & 0.2063 & 0.3968 \\
\hline 843.15 & 0.2774 & 0.3613 & 0.3613 & 0.2639 & 0.3681 & 0.3681 & 0.2494 & 0.3753 & 0.3753 & 0.1989 & 0.4006 & 0.4006 & 0.1790 & 0.4105 \\
\hline 853.15 & 0.2460 & 0.3770 & 0.3770 & 0.2333 & 0.3834 & 0.3834 & 0.2196 & 0.3902 & 0.3902 & 0.1728 & 0.4136 & 0.4136 & 0.1547 & 0.4227 \\
\hline 863.15 & 0.2172 & 0.3914 & 0.3914 & 0.2052 & 0.3974 & 0.3974 & 0.1925 & 0.4037 & 0.4037 & 0.1495 & 0.4252 & 0.4252 & 0.1331 & 0.4334 \\
\hline 873.15 & 0.1908 & 0.4046 & 0.4046 & 0.1797 & 0.4101 & 0.4101 & 0.1681 & 0.4160 & 0.4160 & 0.1290 & 0.4355 & 0.4355 & 0.1143 & 0.4428 \\
\hline 883.15 & 0.1670 & 0.4165 & 0.4165 & 0.1569 & 0.4216 & 0.4216 & 0.1462 & 0.4269 & 0.4269 & 0.1110 & 0.4445 & 0.4445 & 0.0979 & 0.4510 \\
\hline 893.15 & 0.1457 & 0.4271 & 0.4271 & 0.1365 & 0.4318 & 0.4318 & 0.1269 & 0.4366 & 0.4366 & 0.0953 & 0.4523 & 0.4523 & 0.0838 & 0.4581 \\
\hline 903.15 & 0.1268 & 0.4366 & 0.4366 & 0.1185 & 0.4408 & 0.4408 & 0.1098 & 0.4451 & 0.4451 & 0.0818 & 0.4591 & 0.4591 & 0.0717 & 0.4642 \\
\hline 913.15 & 0.1102 & 0.4449 & 0.4449 & 0.1027 & 0.4487 & 0.4487 & 0.0950 & 0.4525 & 0.4525 & 0.0702 & 0.4649 & 0.4649 & 0.0613 & 0.4694 \\
\hline 923.15 & 0.0956 & 0.4522 & 0.4522 & 0.0889 & 0.4556 & 0.4556 & 0.0820 & 0.4590 & 0.4590 & 0.0602 & 0.4699 & 0.4699 & 0.0524 & 0.4738 \\
\hline
\end{tabular}

Table 16. Equilibrium Conversion per Mole Isobutane at Technically Relevant Temperatures and Pressures When 10 Volume Hydrogen is Mixed to 100 Volumes Isobutane Feed.

\begin{tabular}{ccccccc}
\hline & \multicolumn{2}{c}{$0.12 \mathrm{MPa}$} & \multicolumn{2}{c}{$0.11 \mathrm{MPa}$} & \multicolumn{2}{c}{$0.1 \mathrm{MPa}$} \\
\hline$T / \mathrm{K}$ & $X$ & $U_{e}{ }^{a)}$ & $X$ & $U_{e}{ }^{a)}$ & $X$ & $U_{e}{ }^{a)}$ \\
\hline 773.15 & 0.2656 & 0.0353 & 0.2780 & 0.0370 & 0.2921 & 0.0389 \\
783.15 & 0.3012 & 0.0401 & 0.3147 & 0.0419 & 0.3300 & 0.0440 \\
793.15 & 0.3390 & 0.0452 & 0.3535 & 0.0472 & 0.3699 & 0.0494 \\
803.15 & 0.3786 & 0.0506 & 0.3941 & 0.0527 & 0.4115 & 0.0552 \\
813.15 & 0.4194 & 0.0563 & 0.4358 & 0.0586 & 0.4540 & 0.0613 \\
823.15 & 0.4613 & 0.0623 & 0.4782 & 0.0648 & 0.4970 & 0.0676 \\
833.15 & 0.5035 & 0.0685 & 0.5208 & 0.0712 & 0.5399 & 0.0743 \\
843.15 & 0.5454 & 0.0751 & 0.5629 & 0.0779 & 0.5821 & 0.0811 \\
853.15 & 0.5866 & 0.0818 & 0.6040 & 0.0848 & 0.6229 & 0.0881 \\
863.15 & 0.6264 & 0.0887 & 0.6435 & 0.0918 & 0.6619 & 0.0952 \\
873.15 & 0.6644 & 0.0957 & 0.6809 & 0.0989 & 0.6986 & 0.1024 \\
883.15 & 0.7003 & 0.1026 & 0.7160 & 0.1059 & 0.7328 & 0.1095 \\
893.15 & 0.7336 & 0.1096 & 0.7484 & 0.1128 & 0.7642 & 0.1163 \\
903.15 & 0.7642 & 0.1163 & 0.7781 & 0.1195 & 0.7927 & 0.1230 \\
913.15 & 0.7921 & 0.1228 & 0.8049 & 0.1259 & 0.8183 & 0.1292 \\
923.15 & 0.8173 & 0.1289 & 0.8290 & 0.1319 & 0.8412 & 0.1351 \\
\hline \multicolumn{3}{c}{ see Table 14 foot note } & & & &
\end{tabular}

It is clear that to theoretically achieve conversions higher than $90 \%$, the reaction in Catofin process has to be carried at temperatures above $873.15 \mathrm{~K}\left(600{ }^{\circ} \mathrm{C}\right)$ and pressures as low as $0.02 \mathrm{MPa}$. This level of per pass conversion is not achievable with other commercial processes FBD4 and Oleflex. Maximum achievable per pass conversion under $0.12 \mathrm{MPa}$ is $82.6 \pm 10.8 \%$ if to operate under drastic conditions such as at $923.15 \mathrm{~K}$. When operating at $873.15 \mathrm{~K}$ $\left(600{ }^{\circ} \mathrm{C}\right)$ achievable conversion without hydrogen diluent is $68 \pm 8.3 \%$.

Composition of isobutane dehydrogenation reaction mixture at thermodynamic equilibrium was calculated using Eqs. (13) - (16). Results are tabulated in Tables 17 - 24.

\section{Conclusions}

In this study thermodynamic parameters, including molar enthalpy, molar Gibbs free energy, equilibrium constant, conversions and product distribution were calculated for isobutane dehydrogenation reaction at industrially relevant conditions for various processes. The outcome of the study is believed to form a potent source of information for plants operators, researchers and process developers. 
Table 17 - Continued-

\begin{tabular}{c|c|ccc|ccc|ccc|ccc}
\hline & $0.06 \mathrm{MPa}$ & \multicolumn{3}{|c|}{$0.05 \mathrm{MPa}$} & \multicolumn{3}{c|}{$0.04 \mathrm{MPa}$} & \multicolumn{3}{c}{$0.03 \mathrm{MPa}$} & $\boldsymbol{y}^{2}$ \\
\cline { 2 - 11 }$T / \mathrm{K}$ & $\boldsymbol{y}_{H 2}$ & $\boldsymbol{y}_{C 4 H 10}$ & $\boldsymbol{y}_{C 4 H 8}$ & $\boldsymbol{y}_{H 2}$ & $\boldsymbol{y}_{C 4 H 10}$ & $\boldsymbol{y}_{C 4 H 8}$ & $\boldsymbol{y}_{H 2}$ & $\boldsymbol{y}_{C 4 H 10}$ & $\boldsymbol{y}_{C 4 H 8}$ & $\boldsymbol{y}_{H 2}$ & $\boldsymbol{y}_{C 4 H 10}$ & $\boldsymbol{y}_{C 4 H 8}$ & $\boldsymbol{y}_{H 2}$ \\
\hline 773.15 & 0.2871 & 0.3945 & 0.3028 & 0.3028 & 0.3564 & 0.3218 & 0.3218 & 0.3086 & 0.3457 & 0.3457 & 0.2452 & 0.3774 & 0.3774 \\
783.15 & 0.3079 & 0.3532 & 0.3234 & 0.3234 & 0.3160 & 0.3420 & 0.3420 & 0.2699 & 0.3650 & 0.3650 & 0.2105 & 0.3948 & 0.3948 \\
793.15 & 0.3280 & 0.3137 & 0.3432 & 0.3432 & 0.2778 & 0.3611 & 0.3611 & 0.2343 & 0.3829 & 0.3829 & 0.1794 & 0.4103 & 0.4103 \\
803.15 & 0.3472 & 0.2765 & 0.3617 & 0.3617 & 0.2425 & 0.3787 & 0.3787 & 0.2019 & 0.3990 & 0.3990 & 0.1520 & 0.4240 & 0.4240 \\
813.15 & 0.3651 & 0.2422 & 0.3789 & 0.3789 & 0.2104 & 0.3948 & 0.3948 & 0.1730 & 0.4135 & 0.4135 & 0.1282 & 0.4359 & 0.4359 \\
823.15 & 0.3817 & 0.2108 & 0.3946 & 0.3946 & 0.1814 & 0.4093 & 0.4093 & 0.1475 & 0.4262 & 0.4262 & 0.1078 & 0.4461 & 0.4461 \\
833.15 & 0.3968 & 0.1825 & 0.4088 & 0.4088 & 0.1557 & 0.4222 & 0.4222 & 0.1253 & 0.4373 & 0.4373 & 0.0904 & 0.4548 & 0.4548 \\
843.15 & 0.4105 & 0.1572 & 0.4214 & 0.4214 & 0.1331 & 0.4335 & 0.4335 & 0.1061 & 0.4469 & 0.4469 & 0.0757 & 0.4622 & 0.4622 \\
853.15 & 0.4227 & 0.1350 & 0.4325 & 0.4325 & 0.1134 & 0.4433 & 0.4433 & 0.0897 & 0.4552 & 0.4552 & 0.0633 & 0.4684 & 0.4684 \\
863.15 & 0.4334 & 0.1155 & 0.4422 & 0.4422 & 0.0964 & 0.4518 & 0.4518 & 0.0757 & 0.4622 & 0.4622 & 0.0530 & 0.4735 & 0.4735 \\
873.15 & 0.4428 & 0.0987 & 0.4507 & 0.4507 & 0.0819 & 0.4591 & 0.4591 & 0.0639 & 0.4681 & 0.4681 & 0.0444 & 0.4778 & 0.4778 \\
883.15 & 0.4510 & 0.0841 & 0.4579 & 0.4579 & 0.0695 & 0.4653 & 0.4653 & 0.0539 & 0.4731 & 0.4731 & 0.0372 & 0.4814 & 0.4814 \\
893.15 & 0.4581 & 0.0717 & 0.4641 & 0.4641 & 0.0590 & 0.4705 & 0.4705 & 0.0455 & 0.4773 & 0.4773 & 0.0312 & 0.4844 & 0.4844 \\
903.15 & 0.4642 & 0.0611 & 0.4694 & 0.4694 & 0.0500 & 0.4750 & 0.4750 & 0.0385 & 0.4808 & 0.4808 & 0.0263 & 0.4869 & 0.4869 \\
913.15 & 0.4694 & 0.0521 & 0.4740 & 0.4740 & 0.0425 & 0.4787 & 0.4787 & 0.0326 & 0.4837 & 0.4837 & 0.0222 & 0.4889 & 0.4889 \\
923.15 & 0.4738 & 0.0444 & 0.4778 & 0.4778 & 0.0362 & 0.4819 & 0.4819 & 0.0276 & 0.4862 & 0.4862 & 0.0187 & 0.4906 & 0.4906 \\
\hline
\end{tabular}

Table 18. Expanded Uncertainties ${ }^{a}$ in Molar Fractions Reported in Table 17.

\begin{tabular}{|c|c|c|c|c|c|c|c|c|c|c|c|c|c|c|}
\hline \multirow[b]{2}{*}{$T / \mathrm{K}$} & \multicolumn{3}{|c|}{$0.12 \mathrm{MPa}$} & \multicolumn{3}{|c|}{$0.11 \mathrm{MPa}$} & \multicolumn{3}{|c|}{$0.1 \mathrm{MPa}$} & \multicolumn{3}{|c|}{$0.07 \mathrm{MPa}$} & \multicolumn{2}{|c|}{$0.06 \mathrm{MPa}$} \\
\hline & $\boldsymbol{y}_{C 4 H 10}$ & $\boldsymbol{y}_{C 4 H 8}$ & $\boldsymbol{y}_{H 2}$ & $\boldsymbol{y}_{C 4 H 10}$ & $\boldsymbol{y}_{C 4 H 8}$ & $\boldsymbol{y}_{H 2}$ & $\boldsymbol{y}_{C 4 H 10}$ & $\boldsymbol{y}_{C 4 H 8}$ & $\boldsymbol{y}_{H 2}$ & $\boldsymbol{y}_{C 4 H 10}$ & $\boldsymbol{y}_{C 4 H 8}$ & $\boldsymbol{y}_{H 2}$ & $\boldsymbol{y}_{C 4 H 10}$ & $\boldsymbol{y}_{C 4 H 8}$ \\
\hline 773.15 & 0.0152 & 0.0275 & 0.0275 & 0.0313 & 0.0285 & 0.0285 & 0.0322 & 0.0295 & 0.0295 & 0.0355 & 0.0335 & 0.0335 & 0.0369 & 0.0353 \\
\hline 783.15 & 0.0154 & 0.0299 & 0.0299 & 0.0333 & 0.0309 & 0.0309 & 0.0342 & 0.0320 & 0.0320 & 0.0375 & 0.0362 & 0.0362 & 0.0390 & 0.0381 \\
\hline 793.15 & 0.0155 & 0.0324 & 0.0324 & 0.0353 & 0.0334 & 0.0334 & 0.0362 & 0.0345 & 0.0345 & 0.0396 & 0.0389 & 0.0389 & 0.0411 & 0.0409 \\
\hline 803.15 & 0.0155 & 0.0349 & 0.0349 & 0.0372 & 0.0360 & 0.0360 & 0.0382 & 0.0371 & 0.0371 & 0.0417 & 0.0417 & 0.0417 & 0.0433 & 0.0438 \\
\hline 813.15 & 0.0154 & 0.0375 & 0.0375 & 0.0392 & 0.0386 & 0.0386 & 0.0401 & 0.0398 & 0.0398 & 0.0438 & 0.0445 & 0.0445 & 0.0454 & 0.0467 \\
\hline 823.15 & 0.0153 & 0.0400 & 0.0400 & 0.0411 & 0.0412 & 0.0412 & 0.0421 & 0.0424 & 0.0424 & 0.0459 & 0.0474 & 0.0474 & 0.0476 & 0.0496 \\
\hline 833.15 & 0.0152 & 0.0426 & 0.0426 & 0.0431 & 0.0438 & 0.0438 & 0.0441 & 0.0451 & 0.0451 & 0.0480 & 0.0502 & 0.0502 & 0.0498 & 0.0525 \\
\hline 843.15 & 0.0151 & 0.0453 & 0.0453 & 0.0451 & 0.0465 & 0.0465 & 0.0461 & 0.0478 & 0.0478 & 0.0502 & 0.0530 & 0.0530 & 0.0520 & 0.0553 \\
\hline 853.15 & 0.0151 & 0.0479 & 0.0479 & 0.0471 & 0.0491 & 0.0491 & 0.0482 & 0.0505 & 0.0505 & 0.0523 & 0.0558 & 0.0558 & 0.0542 & 0.0581 \\
\hline 863.15 & 0.0153 & 0.0505 & 0.0505 & 0.0491 & 0.0518 & 0.0518 & 0.0502 & 0.0532 & 0.0532 & 0.0545 & 0.0585 & 0.0585 & 0.0563 & 0.0608 \\
\hline 873.15 & 0.0158 & 0.0531 & 0.0531 & 0.0512 & 0.0544 & 0.0544 & 0.0523 & 0.0558 & 0.0558 & 0.0565 & 0.0611 & 0.0611 & 0.0583 & 0.0633 \\
\hline 883.15 & 0.0165 & 0.0557 & 0.0557 & 0.0532 & 0.0570 & 0.0570 & 0.0543 & 0.0584 & 0.0584 & 0.0584 & 0.0636 & 0.0636 & 0.0602 & 0.0657 \\
\hline 893.15 & 0.0176 & 0.0582 & 0.0582 & 0.0551 & 0.0595 & 0.0595 & 0.0562 & 0.0608 & 0.0608 & 0.0602 & 0.0658 & 0.0658 & 0.0619 & 0.0678 \\
\hline 903.15 & 0.0192 & 0.0606 & 0.0606 & 0.0570 & 0.0618 & 0.0618 & 0.0580 & 0.0631 & 0.0631 & 0.0619 & 0.0679 & 0.0679 & 0.0634 & 0.0697 \\
\hline 913.15 & 0.0213 & 0.0628 & 0.0628 & 0.0587 & 0.0640 & 0.0640 & 0.0597 & 0.0653 & 0.0653 & 0.0634 & 0.0697 & 0.0697 & 0.0648 & 0.0714 \\
\hline 923.15 & 0.0240 & 0.0649 & 0.0649 & 0.0603 & 0.0660 & 0.0660 & 0.0613 & 0.0672 & 0.0672 & 0.0647 & 0.0714 & 0.0714 & 0.0660 & 0.0729 \\
\hline
\end{tabular}

a) Calculated based on equations (21) and (22), whereby $f$ is the function between $\mathrm{y}_{\mathrm{i}}$ and $\mathrm{X}$ resulting from substitution $\mathrm{K}$ in equations (12) and (17). Thus resulted equations are the followings:

For isobutane: $\left(\frac{u_{c}(y)}{y}\right)^{2}=\left(\frac{u_{c}(X)}{1-X}\right)^{2}+\left(\frac{u_{c}(X)}{(h+1+X)}\right)^{2}$
For isobutene: $\left(\frac{u_{c}(y)}{y}\right)^{2}=\left(\frac{u_{c}(X)}{X}\right)^{2}+\left(\frac{u_{c}(X)}{(h+1+X)}\right)^{2}$
For hydrogen: $\left(\frac{u_{c}(y)}{y}\right)^{2}=\left(\frac{u_{c}(X)}{(h+1+X)}\right)^{2}+\left(\frac{u_{c}(X)}{(h+1+X)}\right)^{2}$

Table 18 -Continued-

\begin{tabular}{|c|c|c|c|c|c|c|c|c|c|c|c|c|c|}
\hline \multirow[b]{2}{*}{$T / \mathrm{K}$} & \multirow{2}{*}{$\begin{array}{c}0.06 \mathrm{MPa} \\
\boldsymbol{y}_{H 2}\end{array}$} & \multicolumn{3}{|c|}{$0.05 \mathrm{MPa}$} & \multicolumn{3}{|c|}{$0.04 \mathrm{MPa}$} & \multicolumn{3}{|c|}{$0.03 \mathrm{MPa}$} & \multicolumn{3}{|c|}{$0.02 \mathrm{MPa}$} \\
\hline & & $\boldsymbol{y}_{C 4 H 10}$ & $\boldsymbol{y}_{C 4 H 8}$ & $\boldsymbol{y}_{H 2}$ & $\boldsymbol{y}_{C 4 H 10}$ & $\boldsymbol{y}_{C 4 H 8}$ & $\boldsymbol{y}_{\mathrm{H} 2}$ & $\boldsymbol{y}_{C 4 H 10}$ & $\boldsymbol{y}_{C 4 H 8}$ & $\boldsymbol{y}_{\mathrm{H} 2}$ & $\boldsymbol{y}_{C 4 H 10}$ & $\boldsymbol{y}_{C 4 H 8}$ & $\boldsymbol{y}_{H 2}$ \\
\hline 773.15 & 0.0353 & 0.0387 & 0.0376 & 0.0376 & 0.0409 & 0.0405 & 0.0405 & 0.0439 & 0.0443 & 0.0443 & 0.0483 & 0.0502 & 0.0502 \\
\hline 783.15 & 0.0381 & 0.0408 & 0.0405 & 0.0405 & 0.0431 & 0.0435 & 0.0435 & 0.0462 & 0.0475 & 0.0475 & 0.0509 & 0.0535 & 0.0535 \\
\hline 793.15 & 0.0409 & 0.0430 & 0.0434 & 0.0434 & 0.0454 & 0.0465 & 0.0465 & 0.0486 & 0.0507 & 0.0507 & 0.0534 & 0.0568 & 0.0568 \\
\hline 803.15 & 0.0438 & 0.0452 & 0.0463 & 0.0463 & 0.0477 & 0.0496 & 0.0496 & 0.0510 & 0.0539 & 0.0539 & 0.0559 & 0.0601 & 0.0601 \\
\hline 813.15 & 0.0467 & 0.0474 & 0.0493 & 0.0493 & 0.0500 & 0.0526 & 0.0526 & 0.0534 & 0.0570 & 0.0570 & 0.0584 & 0.0632 & 0.0632 \\
\hline 823.15 & 0.0496 & 0.0497 & 0.0523 & 0.0523 & 0.0523 & 0.0556 & 0.0556 & 0.0558 & 0.0600 & 0.0600 & 0.0607 & 0.0660 & 0.0660 \\
\hline 833.15 & 0.0525 & 0.0520 & 0.0552 & 0.0552 & 0.0546 & 0.0586 & 0.0586 & 0.0581 & 0.0629 & 0.0629 & 0.0628 & 0.0687 & 0.0687 \\
\hline 843.15 & 0.0553 & 0.0542 & 0.0581 & 0.0581 & 0.0569 & 0.0614 & 0.0614 & 0.0602 & 0.0656 & 0.0656 & 0.0647 & 0.0710 & 0.0710 \\
\hline 853.15 & 0.0581 & 0.0563 & 0.0608 & 0.0608 & 0.0590 & 0.0641 & 0.0641 & 0.0622 & 0.0681 & 0.0681 & 0.0663 & 0.0731 & 0.0731 \\
\hline 863.15 & 0.0608 & 0.0584 & 0.0634 & 0.0634 & 0.0609 & 0.0666 & 0.0666 & 0.0640 & 0.0703 & 0.0703 & 0.0677 & 0.0749 & 0.0749 \\
\hline 873.15 & 0.0633 & 0.0603 & 0.0659 & 0.0659 & 0.0627 & 0.0688 & 0.0688 & 0.0656 & 0.0723 & 0.0723 & 0.0690 & 0.0764 & 0.0764 \\
\hline 883.15 & 0.0657 & 0.0621 & 0.0681 & 0.0681 & 0.0644 & 0.0708 & 0.0708 & 0.0669 & 0.0739 & 0.0739 & 0.0700 & 0.0776 & 0.0776 \\
\hline 893.15 & 0.0678 & 0.0637 & 0.0701 & 0.0701 & 0.0658 & 0.0726 & 0.0726 & 0.0681 & 0.0754 & 0.0754 & 0.0708 & 0.0786 & 0.0786 \\
\hline 903.15 & 0.0697 & 0.0651 & 0.0718 & 0.0718 & 0.0670 & 0.0741 & 0.0741 & 0.0691 & 0.0766 & 0.0766 & 0.0714 & 0.0794 & 0.0794 \\
\hline 913.15 & 0.0714 & 0.0663 & 0.0733 & 0.0733 & 0.0680 & 0.0754 & 0.0754 & 0.0699 & 0.0776 & 0.0776 & 0.0719 & 0.0801 & 0.0801 \\
\hline 923.15 & 0.0729 & 0.0674 & 0.0746 & 0.0746 & 0.0689 & 0.0764 & 0.0764 & 0.0706 & 0.0784 & 0.0784 & 0.0723 & 0.0806 & 0.0806 \\
\hline
\end{tabular}


Table 19. Molar Fractions of Reaction Species in Equilibrium of Isobutane Dehydrogenation to Isobutene Reaction at Technically Relevant Temperatures and Pressures when 1 Volume Hydrogen Is Mixed To 100 Volumes Isobutane Feed, Uncertainties are Given in Table 20.

\begin{tabular}{|c|c|c|c|c|c|c|c|c|c|}
\hline \multirow{2}{*}{$T / \mathrm{K}$} & \multicolumn{3}{|c|}{$0.12 \mathrm{MPa}$} & \multicolumn{3}{|c|}{$0.11 \mathrm{MPa}$} & \multicolumn{3}{|c|}{$0.1 \mathrm{MPa}$} \\
\hline & $\boldsymbol{y}_{C 4 H 10}$ & $\boldsymbol{y}_{C 4 H 8}$ & $\boldsymbol{y}_{H 2}$ & $\boldsymbol{y}_{C 4 H 10}$ & $\boldsymbol{y}_{C 4 H 8}$ & $y_{H 2}$ & $\boldsymbol{y}_{C 4 H 10}$ & $\boldsymbol{y}_{C 4 H 8}$ & $\boldsymbol{y}_{H 2}$ \\
\hline 773.15 & 0.2329 & 0.2253 & 0.5418 & 0.2399 & 0.2323 & 0.5277 & 0.2477 & 0.2402 & 0.5121 \\
\hline 783.15 & 0.2527 & 0.2452 & 0.5021 & 0.2599 & 0.2525 & 0.4875 & 0.2679 & 0.2606 & 0.4715 \\
\hline 793.15 & 0.2725 & 0.2653 & 0.4622 & 0.2799 & 0.2727 & 0.4474 & 0.2880 & 0.2809 & 0.4311 \\
\hline 803.15 & 0.2922 & 0.2851 & 0.4226 & 0.2997 & 0.2926 & 0.4077 & 0.3078 & 0.3009 & 0.3914 \\
\hline 813.15 & 0.3114 & 0.3046 & 0.3840 & 0.3188 & 0.3120 & 0.3692 & 0.3269 & 0.3201 & 0.3530 \\
\hline 823.15 & 0.3300 & 0.3233 & 0.3466 & 0.3373 & 0.3306 & 0.3321 & 0.3451 & 0.3386 & 0.3163 \\
\hline 833.15 & 0.3478 & 0.3412 & 0.3110 & 0.3548 & 0.3483 & 0.2969 & 0.3623 & 0.3560 & 0.2817 \\
\hline 843.15 & 0.3645 & 0.3581 & 0.2774 & 0.3712 & 0.3649 & 0.2639 & 0.3784 & 0.3722 & 0.2494 \\
\hline 853.15 & 0.3801 & 0.3739 & 0.2460 & 0.3864 & 0.3803 & 0.2332 & 0.3932 & 0.3871 & 0.2196 \\
\hline 863.15 & 0.3945 & 0.3884 & 0.2172 & 0.4004 & 0.3944 & 0.2052 & 0.4067 & 0.4008 & 0.1925 \\
\hline 873.15 & 0.4076 & 0.4016 & 0.1908 & 0.4131 & 0.4072 & 0.1797 & 0.4189 & 0.4131 & 0.1681 \\
\hline 883.15 & 0.4194 & 0.4136 & 0.1670 & 0.4244 & 0.4187 & 0.1569 & 0.4297 & 0.4240 & 0.1462 \\
\hline 893.15 & 0.4300 & 0.4243 & 0.1457 & 0.4346 & 0.4289 & 0.1365 & 0.4394 & 0.4338 & 0.1269 \\
\hline 903.15 & 0.4394 & 0.4338 & 0.1268 & 0.4435 & 0.4380 & 0.1185 & 0.4478 & 0.4423 & 0.1098 \\
\hline 913.15 & 0.4477 & 0.4422 & 0.1102 & 0.4514 & 0.4459 & 0.1027 & 0.4552 & 0.4498 & 0.0950 \\
\hline 923.15 & 0.4549 & 0.4495 & 0.0956 & 0.4583 & 0.4528 & 0.0889 & 0.4617 & 0.4563 & 0.0820 \\
\hline
\end{tabular}

Table 20. Expanded Uncertainties ${ }^{a}$ in Molar Fractions Reported in Table 19.

\begin{tabular}{|c|c|c|c|c|c|c|c|c|c|}
\hline \multirow[b]{2}{*}{$T / \mathrm{K}$} & \multicolumn{3}{|c|}{$0.12 \mathrm{MPa}$} & \multicolumn{3}{|c|}{$0.11 \mathrm{MPa}$} & \multicolumn{3}{|c|}{$0.1 \mathrm{MPa}$} \\
\hline & $\boldsymbol{y}_{C 4 H 10}$ & $\boldsymbol{y}_{C 4 H 8}$ & $\boldsymbol{y}_{H 2}$ & $\boldsymbol{y}_{C 4 H 10}$ & $\boldsymbol{y}_{C 4 H 8}$ & $\boldsymbol{y}_{H 2}$ & $\boldsymbol{y}_{C 4 H 10}$ & $\boldsymbol{y}_{C 4 H 8}$ & $\boldsymbol{y}_{H 2}$ \\
\hline 773.15 & 0.0341 & 0.0307 & 0.0308 & 0.0350 & 0.0317 & 0.0318 & 0.0359 & 0.0329 & 0.0329 \\
\hline 783.15 & 0.0365 & 0.0336 & 0.0337 & 0.0374 & 0.0347 & 0.0347 & 0.0383 & 0.0358 & 0.0359 \\
\hline 793.15 & 0.0389 & 0.0366 & 0.0366 & 0.0398 & 0.0377 & 0.0377 & 0.0408 & 0.0389 & 0.0389 \\
\hline 803.15 & 0.0413 & 0.0396 & 0.0397 & 0.0422 & 0.0407 & 0.0408 & 0.0432 & 0.0420 & 0.0420 \\
\hline 813.15 & 0.0438 & 0.0427 & 0.0428 & 0.0446 & 0.0438 & 0.0439 & 0.0455 & 0.0451 & 0.0452 \\
\hline 823.15 & 0.0462 & 0.0459 & 0.0460 & 0.0470 & 0.0470 & 0.0471 & 0.0479 & 0.0483 & 0.0483 \\
\hline 833.15 & 0.0486 & 0.0491 & 0.0492 & 0.0495 & 0.0502 & 0.0503 & 0.0503 & 0.0514 & 0.0515 \\
\hline 843.15 & 0.0511 & 0.0523 & 0.0525 & 0.0519 & 0.0535 & 0.0536 & 0.0528 & 0.0547 & 0.0548 \\
\hline 853.15 & 0.0537 & 0.0557 & 0.0558 & 0.0544 & 0.0567 & 0.0568 & 0.0553 & 0.0579 & 0.0580 \\
\hline 863.15 & 0.0563 & 0.0590 & 0.0591 & 0.0570 & 0.0600 & 0.0601 & 0.0578 & 0.0611 & 0.0612 \\
\hline 873.15 & 0.0589 & 0.0623 & 0.0624 & 0.0596 & 0.0633 & 0.0634 & 0.0603 & 0.0643 & 0.0645 \\
\hline 883.15 & 0.0615 & 0.0656 & 0.0657 & 0.0621 & 0.0665 & 0.0667 & 0.0628 & 0.0675 & 0.0676 \\
\hline 893.15 & 0.0640 & 0.0688 & 0.0690 & 0.0646 & 0.0697 & 0.0698 & 0.0653 & 0.0706 & 0.0708 \\
\hline 903.15 & 0.0665 & 0.0719 & 0.0721 & 0.0671 & 0.0728 & 0.0729 & 0.0677 & 0.0736 & 0.0737 \\
\hline 913.15 & 0.0689 & 0.0749 & 0.0751 & 0.0695 & 0.0757 & 0.0758 & 0.0700 & 0.0764 & 0.0766 \\
\hline 923.15 & 0.0712 & 0.0777 & 0.0779 & 0.0717 & 0.0784 & 0.0786 & 0.0722 & 0.0791 & 0.0793 \\
\hline
\end{tabular}

a) Calculated based on equations (21) and (33 through 35)

Table 21. Molar Fractions of Reaction Species in Equilibrium of Isobutane Dehydrogenation to Isobutene Reaction at Technically Relevant Temperatures and Pressures When 5 Volume Hydrogen is Mixed to 100 Volumes Isobutane Feed, Uncertainties are Given in Table 22.

\begin{tabular}{c|lll|lll|llr}
\hline \multirow{2}{*}{$T / \mathrm{K}$} & \multicolumn{3}{|c|}{$0.12 \mathrm{MPa}$} & \multicolumn{3}{c|}{$0.11 \mathrm{MPa}$} & \multicolumn{3}{c}{$0.1 \mathrm{MPa}$} \\
\cline { 2 - 9 } & $\boldsymbol{y}_{C 4 H 10}$ & $\boldsymbol{y}_{C 4 H 8}$ & $\boldsymbol{y}_{H 2}$ & $\boldsymbol{y}_{C 4 H 10}$ & $\boldsymbol{y}_{C 4 H 8}$ & $\boldsymbol{y}_{H 2}$ & $\boldsymbol{y}_{C 4 H 10}$ & $\boldsymbol{y}_{C 4 H 8}$ & $\boldsymbol{y}_{H 2}$ \\
\hline 773.15 & 0.2484 & 0.2108 & 0.5408 & 0.2552 & 0.2180 & 0.5268 & 0.2628 & 0.2260 & 0.5112 \\
783.15 & 0.2677 & 0.2311 & 0.5012 & 0.2748 & 0.2385 & 0.4867 & 0.2826 & 0.2467 & 0.4707 \\
793.15 & 0.2871 & 0.2514 & 0.4615 & 0.2943 & 0.2590 & 0.4467 & 0.3022 & 0.2673 & 0.4304 \\
803.15 & 0.3063 & 0.2716 & 0.4220 & 0.3136 & 0.2793 & 0.4071 & 0.3215 & 0.2876 & 0.3909 \\
813.15 & 0.3251 & 0.2914 & 0.3835 & 0.3323 & 0.2990 & 0.3687 & 0.3402 & 0.3072 & 0.3526 \\
823.15 & 0.3433 & 0.3105 & 0.3462 & 0.3504 & 0.3179 & 0.3317 & 0.3581 & 0.3260 & 0.3160 \\
833.15 & 0.3607 & 0.3287 & 0.3107 & 0.3675 & 0.3359 & 0.2966 & 0.3749 & 0.3437 & 0.2814 \\
843.15 & 0.3770 & 0.3459 & 0.2771 & 0.3836 & 0.3528 & 0.2636 & 0.3906 & 0.3602 & 0.2492 \\
853.15 & 0.3923 & 0.3619 & 0.2458 & 0.3985 & 0.3684 & 0.2330 & 0.4051 & 0.3754 & 0.2194 \\
863.15 & 0.4064 & 0.3767 & 0.2170 & 0.4122 & 0.3828 & 0.2050 & 0.4184 & 0.3893 & 0.1924 \\
873.15 & 0.4192 & 0.3902 & 0.1907 & 0.4246 & 0.3958 & 0.1796 & 0.4303 & 0.4018 & 0.1679 \\
883.15 & 0.4308 & 0.4023 & 0.1669 & 0.4357 & 0.4075 & 0.1567 & 0.4409 & 0.4130 & 0.1461 \\
893.15 & 0.4412 & 0.4132 & 0.1456 & 0.4457 & 0.4179 & 0.1364 & 0.4504 & 0.4229 & 0.1268 \\
903.15 & 0.4504 & 0.4229 & 0.1267 & 0.4544 & 0.4272 & 0.1184 & 0.4586 & 0.4316 & 0.1098 \\
913.15 & 0.4585 & 0.4314 & 0.1101 & 0.4621 & 0.4352 & 0.1026 & 0.4659 & 0.4392 & 0.0949 \\
923.15 & 0.4656 & 0.4389 & 0.0955 & 0.4689 & 0.4423 & 0.0888 & 0.4722 & 0.4458 & 0.0820 \\
\hline
\end{tabular}


Table 22. Expanded Uncertainties ${ }^{a}$ in Molar Fractions Reported in Table 21.

\begin{tabular}{ccccccccrr}
\hline \multirow{2}{*}{$T / \mathrm{K}$} & \multicolumn{3}{c}{$0.12 \mathrm{MPa}$} & \multicolumn{3}{c}{$0.11 \mathrm{MPa}$} & \multicolumn{3}{c}{$0.1 \mathrm{MPa}$} \\
\cline { 2 - 9 } & $\boldsymbol{y}_{C 4 H 10}$ & $\boldsymbol{y}_{C 4 H 8}$ & $\boldsymbol{y}_{H 2}$ & $\boldsymbol{y}_{C 4 H 10}$ & $\boldsymbol{y}_{C 4 H 8}$ & $\boldsymbol{y}_{H 2}$ & $\boldsymbol{y}_{C 4 H 10}$ & $\boldsymbol{y}_{C 4 H 8}$ & $\boldsymbol{y}_{H 2}$ \\
\hline 773.15 & 0.0319 & 0.0287 & 0.0289 & 0.0328 & 0.0297 & 0.0300 & 0.0338 & 0.0309 & 0.0311 \\
783.15 & 0.0344 & 0.0316 & 0.0318 & 0.0353 & 0.0327 & 0.0329 & 0.0364 & 0.0339 & 0.0342 \\
793.15 & 0.0369 & 0.0345 & 0.0349 & 0.0378 & 0.0357 & 0.0360 & 0.0389 & 0.0370 & 0.0373 \\
803.15 & 0.0394 & 0.0376 & 0.0379 & 0.0404 & 0.0388 & 0.0392 & 0.0414 & 0.0402 & 0.0405 \\
813.15 & 0.0419 & 0.0407 & 0.0411 & 0.0429 & 0.0420 & 0.0424 & 0.0440 & 0.0434 & 0.0438 \\
823.15 & 0.0443 & 0.0439 & 0.0443 & 0.0454 & 0.0452 & 0.0456 & 0.0465 & 0.0467 & 0.0471 \\
833.15 & 0.0469 & 0.0471 & 0.0476 & 0.0479 & 0.0485 & 0.0489 & 0.0491 & 0.0500 & 0.0505 \\
843.15 & 0.0494 & 0.0504 & 0.0509 & 0.0505 & 0.0518 & 0.0523 & 0.0517 & 0.0533 & 0.0539 \\
853.15 & 0.0520 & 0.0537 & 0.0542 & 0.0531 & 0.0551 & 0.0557 & 0.0544 & 0.0567 & 0.0573 \\
863.15 & 0.0546 & 0.0570 & 0.0576 & 0.0557 & 0.0585 & 0.0591 & 0.0570 & 0.0601 & 0.0607 \\
873.15 & 0.0572 & 0.0603 & 0.0609 & 0.0584 & 0.0618 & 0.0624 & 0.0597 & 0.0635 & 0.0641 \\
883.15 & 0.0598 & 0.0636 & 0.0642 & 0.0610 & 0.0651 & 0.0657 & 0.0623 & 0.0667 & 0.0674 \\
893.15 & 0.0624 & 0.0668 & 0.0674 & 0.0636 & 0.0683 & 0.0690 & 0.0649 & 0.0699 & 0.0706 \\
903.15 & 0.0649 & 0.0699 & 0.0706 & 0.0661 & 0.0713 & 0.0721 & 0.0673 & 0.0729 & 0.0737 \\
913.15 & 0.0673 & 0.0728 & 0.0736 & 0.0684 & 0.0742 & 0.0750 & 0.0697 & 0.0758 & 0.0765 \\
923.15 & 0.0696 & 0.0756 & 0.0764 & 0.0707 & 0.0772 & 0.0777 & 0.0718 & 0.0787 & 0.0792 \\
\hline a) Calculated based on equations (21) and (33 through 35) & & & & &
\end{tabular}

Table 23. Molar Fractions of Reaction Species in Equilibrium of Isobutane Dehydrogenation to Isobutene Reaction at Technically Relevant Temperatures and Pressures When 10 Volume Hydrogen is Mixed to 100 Volumes Isobutane Feed, Uncertainties are Given in Table 24.

\begin{tabular}{c|ccc|ccc|ccc}
\hline \multirow{2}{*}{$T / \mathrm{K}$} & \multicolumn{3}{|c|}{$0.12 \mathrm{MPa}$} & \multicolumn{3}{c|}{$0.11 \mathrm{MPa}$} & \multicolumn{3}{c}{$0.1 \mathrm{MPa}$} \\
\cline { 2 - 9 } & $\boldsymbol{y}_{C 4 H 10}$ & $\boldsymbol{y}_{C 4 H 8}$ & $\boldsymbol{y}_{H 2}$ & $\boldsymbol{y}_{C 4 H 10}$ & $\boldsymbol{y}_{C 4 H 8}$ & $\boldsymbol{y}_{H 2}$ & $\boldsymbol{y}_{C 4 H 10}$ & $\boldsymbol{y}_{C 4 H 8}$ & $\boldsymbol{y}_{H 2}$ \\
\hline 773.15 & 0.2677 & 0.1945 & 0.5378 & 0.2743 & 0.2018 & 0.5239 & 0.2816 & 0.2098 & 0.5085 \\
783.15 & 0.2863 & 0.2150 & 0.4987 & 0.2932 & 0.2225 & 0.4844 & 0.3007 & 0.2308 & 0.4685 \\
793.15 & 0.3051 & 0.2356 & 0.4594 & 0.3120 & 0.2432 & 0.4448 & 0.3197 & 0.2517 & 0.4286 \\
803.15 & 0.3237 & 0.2560 & 0.4203 & 0.3307 & 0.2638 & 0.4055 & 0.3384 & 0.2722 & 0.3894 \\
813.15 & 0.3419 & 0.2761 & 0.3821 & 0.3489 & 0.2838 & 0.3674 & 0.3565 & 0.2921 & 0.3514 \\
823.15 & 0.3595 & 0.2955 & 0.3450 & 0.3664 & 0.3030 & 0.3306 & 0.3738 & 0.3112 & 0.3150 \\
833.15 & 0.3763 & 0.3140 & 0.3097 & 0.3830 & 0.3213 & 0.2957 & 0.3902 & 0.3292 & 0.2806 \\
843.15 & 0.3922 & 0.3315 & 0.2763 & 0.3986 & 0.3385 & 0.2629 & 0.4055 & 0.3460 & 0.2485 \\
853.15 & 0.4071 & 0.3478 & 0.2451 & 0.4131 & 0.3545 & 0.2324 & 0.4196 & 0.3615 & 0.2189 \\
863.15 & 0.4208 & 0.3628 & 0.2164 & 0.4264 & 0.3691 & 0.2045 & 0.4324 & 0.3757 & 0.1919 \\
873.15 & 0.4332 & 0.3766 & 0.1902 & 0.4385 & 0.3823 & 0.1792 & 0.4440 & 0.3884 & 0.1675 \\
883.15 & 0.4445 & 0.3890 & 0.1665 & 0.4493 & 0.3943 & 0.1564 & 0.4544 & 0.3998 & 0.1458 \\
893.15 & 0.4546 & 0.4001 & 0.1453 & 0.4590 & 0.4049 & 0.1361 & 0.4636 & 0.4099 & 0.1265 \\
903.15 & 0.4636 & 0.4099 & 0.1265 & 0.4675 & 0.4143 & 0.1182 & 0.4716 & 0.4188 & 0.1095 \\
913.15 & 0.4715 & 0.4186 & 0.1099 & 0.4750 & 0.4226 & 0.1024 & 0.4787 & 0.4266 & 0.0947 \\
923.15 & 0.4784 & 0.4263 & 0.0953 & 0.4816 & 0.4298 & 0.0887 & 0.4849 & 0.4333 & 0.0818 \\
\hline
\end{tabular}

Table 24. Expanded Uncertainties ${ }^{a}$ in Molar Fractions Reported in Table 23.

\begin{tabular}{rlllllrrrr}
\hline \multirow{2}{*}{$T / \mathrm{K}$} & \multicolumn{3}{c}{$0.12 \mathrm{MPa}$} & \multicolumn{3}{c}{$0.11 \mathrm{MPa}$} & \multicolumn{3}{c}{$0.1 \mathrm{MPa}$} \\
\cline { 2 - 9 } & $\boldsymbol{y}_{C 4 H 10}$ & $\boldsymbol{y}_{C 4 H 8}$ & $\boldsymbol{y}_{H 2}$ & $\boldsymbol{y}_{C 4 H 10}$ & $\boldsymbol{y}_{C 4 H 8}$ & $\boldsymbol{y}_{H 2}$ & $\boldsymbol{y}_{C 4 H 10}$ & $\boldsymbol{y}_{C 4 H 8}$ & $\boldsymbol{y}_{H 2}$ \\
\hline 773.15 & 0.0294 & 0.0264 & 0.0268 & 0.0303 & 0.0274 & 0.0279 & 0.0314 & 0.0286 & 0.0290 \\
783.15 & 0.0320 & 0.0293 & 0.0298 & 0.0329 & 0.0304 & 0.0309 & 0.0340 & 0.0316 & 0.0321 \\
793.15 & 0.0345 & 0.0322 & 0.0328 & 0.0355 & 0.0334 & 0.0340 & 0.0366 & 0.0347 & 0.0353 \\
803.15 & 0.0371 & 0.0353 & 0.0359 & 0.0381 & 0.0365 & 0.0372 & 0.0392 & 0.0379 & 0.0386 \\
813.15 & 0.0396 & 0.0384 & 0.0391 & 0.0407 & 0.0397 & 0.0404 & 0.0418 & 0.0411 & 0.0419 \\
823.15 & 0.0422 & 0.0416 & 0.0424 & 0.0432 & 0.0429 & 0.0437 & 0.0444 & 0.0444 & 0.0452 \\
833.15 & 0.0447 & 0.0448 & 0.0457 & 0.0458 & 0.0462 & 0.0471 & 0.0470 & 0.0477 & 0.0486 \\
843.15 & 0.0473 & 0.0481 & 0.0490 & 0.0484 & 0.0495 & 0.0504 & 0.0497 & 0.0510 & 0.0520 \\
853.15 & 0.0499 & 0.0513 & 0.0524 & 0.0511 & 0.0528 & 0.0538 & 0.0524 & 0.0544 & 0.0555 \\
863.15 & 0.0526 & 0.0546 & 0.0557 & 0.0537 & 0.0561 & 0.0572 & 0.0550 & 0.0577 & 0.0589 \\
873.15 & 0.0552 & 0.0579 & 0.0591 & 0.0564 & 0.0594 & 0.0606 & 0.0577 & 0.0611 & 0.0623 \\
883.15 & 0.0578 & 0.0612 & 0.0624 & 0.0590 & 0.0627 & 0.0639 & 0.0603 & 0.0643 & 0.0656 \\
893.15 & 0.0604 & 0.0644 & 0.0656 & 0.0616 & 0.0658 & 0.0671 & 0.0629 & 0.0674 & 0.0688 \\
903.15 & 0.0629 & 0.0674 & 0.0688 & 0.0641 & 0.0689 & 0.0702 & 0.0654 & 0.0704 & 0.0718 \\
913.15 & 0.0653 & 0.0703 & 0.0717 & 0.0664 & 0.0717 & 0.0732 & 0.0677 & 0.0732 & 0.0747 \\
923.15 & 0.0675 & 0.0731 & 0.0745 & 0.0687 & 0.0744 & 0.0759 & 0.0698 & 0.0759 & 0.0773 \\
\hline
\end{tabular}

a) Calculated based on equations (21) and (33 through 35)

\section{Nomenclature}

$a_{n, i} \quad$ Gas phase heat capacity constants $\left(a_{0, i}, a_{1, i}, a_{2, i}\right.$, $\left.a_{3, i}, a_{4, i}\right)$ of the species $i$, in $\mathrm{J} \mathrm{mol}^{-1} \mathrm{~K}^{-1}$

$\Delta_{R} a_{n} \quad$ Reaction mixture gas phase heat capacity constants $\left(\Delta_{R} a_{0}, \Delta_{R} a_{1}, \Delta_{R} a_{2}, \Delta_{R} a_{3}\right)$, in $\mathrm{J} \mathrm{mol}^{-1} \mathrm{~K}^{-1}$
$C_{p_{T}, i}^{o} \quad$ Ideal gas heat capacity of species $i$ at $T$, in $\mathrm{J} \mathrm{mol}^{-1} \mathrm{~K}^{-1}$ $\Delta_{R} C_{p_{T}}^{o} \quad$ Reaction mixture heat capacity at $T$

$\Delta H_{f, i}^{o} \quad$ Ideal gas standard enthalpy of formation

$\varepsilon \quad$ Reaction number

$\Delta G_{f, i}^{o} \quad$ Ideal gas standard free enthalpy of formation 
$\Delta_{\mathrm{R}} G_{T}^{o} \quad$ Reaction free Gibbs energy at temperature $T$

$\Delta_{\mathrm{R}} H_{T}^{o} \quad$ Reaction enthalpy at temperature $T$

$K \quad$ Equilibrium constant

$K_{T} \quad$ Equilibrium constant at temperature $T$

$\dot{n}_{i} \quad$ Mole stream of species i out of reactor

$\dot{n}_{i, o} \quad$ Mole stream of species $i$ in the feed

$v_{\mathrm{i}} \quad$ Stoichiometric number of moles of species $i$

$v \quad$ Reaction stoichiometric number

$P \quad$ Reaction pressure, bar

$P^{o} \quad$ Standard pressure, bar

$R \quad$ Ideal gas constant, ( $8.314 \mathrm{~J} \mathrm{~mol}^{-1} \mathrm{~K}^{-1}$ )

$S_{f, i}^{o} \quad$ Ideal gas standard entropy of formation

$\Delta_{\mathrm{R}} S_{T}^{o} \quad$ Reaction entropy at temperature $T$

$T \quad$ Temperature, in Kelvin

$u_{e}(x) \quad$ Expanded uncertainty in the variable $x$

$u_{r}(x) \quad$ Relative uncertainty in the variable $x$

$X \quad$ Conversion, per mole isobutane

$\mathrm{y}_{\mathrm{i}} \quad$ Mole fraction of species $i$

\section{References}

[1] D. Sanfilippo, I. Miracca, F. Trifirò, Dehydrogenation Processes, In Encyclopedia of Hydrocarbons, Grafiche, M. Ed. Roma-Italy: S.P.A., 2005.

[2] D. E. Resasco, Dehydrogenation by Heterogeneous Catalysts, In Encyclopedia of Catalysis, Horvath, I.T.N.Y., Ed. New York: Wiley, 2003.

[3] B. M. Weckhuysen, R. A. Schoonheydt, "Alkane dehydrogenation over supported chromium oxide catalysts," Catalysis Today, 51, 223-232, 1999.

[4] J. Gregor, D. Wei, UOP Oleflex Process for light olefin production, In Handbook of Petrochemicals Production Processes, Meyers, R. A., Ed. New York: McGraw-Hill, 2005.

[5] H. Liu, Z. Yu, A. Ma, Y. Sun, Z. Wang, "Thermodynamic analysis on dehydrogenation of isobutane to isobutene," Acta Petrolei Sinica, 27, 419423, 2011. (Chinese)

[6] L. Constantinou, and R. Gani. "New group contribution method for estimating properties of pure compounds," AIChE J, 40, 1697-1710, 1994.

[7] E. S. Domalski, E. D. Hearing, "Estimation of the thermodynamic properties of hydrocarbons at $298.15 \mathrm{~K}$," J. Phys. Chem. Ref. Data, 17, 1637-1678, 1988.

[8] R. A. Alberty, C. A. Gehrig, "Standard chemical thermodynamic properties of alkene isomer groups," $J$. Phys. Chem. Ref. data, 14, 803-820, 1985.

[9] Joback, K. G. (1984). A Unified Approach to Physical Property Estimation Using Multivariate Statistical Techniques (MS. Thesis), Massachusetts Institute of Technology, Cambridge, MA.

[10] J. Happel, R. Mezaki, "Thermodynamic equilibrium constant of isobutane-isobutene-hydrogen system," $J$. Chem. Eng. Data, 18, 2, 152-154, 1973.

[11] J. E. Germain, Catalytic Conversion of Hydrocarbons, London: Academic Press, 1969.

[12] S. W. Benson, F. R. Cruickshank, D. M. Golden, G. R. Haugen, H. E. O’Neal, A. S. Rodgers, R. Shaw, R. Walsh, "Additivity rules for the estimation of thermochemical properties," Chem. Rev., 69, 279-324, 1969.
[13] B. E. Poling, J. M. Prausnitz, J. P. O' Connell, The Properties of Gases and Liquids, $5^{\text {th }}$ Ed. New York: McGraw-Hill, 2001

[14] N. Cohen, S. W. Benson, Alkanes and Cycloalkanes, West Sussex, England: John Wiley and Sons Ltd., doi: 10.1002/0470034378. ch6. 1992. Published Online: 8 AUG 2006

[15] J. E. Kilpatrick, K. S. Pitzer, "Heat Content, Freeenergy Function, Entropy, and Heat Capacity of Ethylene, Propylene, and the Four Butenes to 1,500K," Journal of Research, U. S. Department of Commerce National Bureau of Standards, Research Paper RP 1738, Vol. 37, 163-171, September 1946

[16] JCGM 100:2008, Evaluation of measurement data Guide to the expression of uncertainty in measurement, Document produced by Working Group (JCGM/WG 1) 2008, Corrected version 2010.

[17] S. W. Benson, Thermochemical Kinetics, $2^{\text {nd }}$ Ed. New York: Wiley, 1976.

[18] R. C. Reid, J. M. Prausnitz, B. E. Poling, The Properties of Gases and Liquids, $4^{\text {th }}$ Ed. New York: McGraw-Hill, 1987.

[19] R. L. Rowley, W. V. Wilding, J. L. Oscarson, Y. Yang, N. A. Zundel, T. E. Daubert, R. P. Danner. "DIPPR ${ }^{\circledR}$. Data Compilation of Pure Chemical Properties, Design Institute for Physical Properties," AIChE, New York, 2007

[20] G. Kyle, Chemical and Process Thermodynamics, Engelwood Cliffs, NJ: Prentice-Hall, 1984.

[21] M. W. Chase, "Jr., NIST-JANAF Thermo-chemical Tables, 4 ", Journal of Physical Chemical Reference Data, Monograph 9, 1-1951, 1998.

[22] F. A. Aly, L.L. Lee, "Self-consistent equations for calculating the ideal-gas heat capacity, enthalpy and entropy," Fluid Phase Equilibria, 6, 69-79, 1981.

[23] S. S. Chen, "Ideal gas thermodynamic properties and isomerization of n-butane and isobutane," J. Phys. Chem. Ref. Data, 4, 859-869, 1975.

[24] K. Wark, Thermodynamics, $4^{\text {th }} E d$. New York: Mc Graw-Hill, 1983.

[25] E. J. Prosen, E. W. Maron, F. Rossini, "Heats of combustion, formation, and isomerization of ten $\mathrm{c} 4$ hydrocarbons", J. Res. Natl. Bur. Stand. (U.S.), 46, 106, 1951.

[26] D. W. Scott, "The chemical thermodynamic properties of hydrocarbons and related substances," Washington U.S. Bureau of Mines, Bull. 666, p187, 1974.

[27] J. B. Pedley, R. D. Naylor, S. P. Kirby, Thermochemical Data of Organic Compounds, $2^{\text {nd }} E d$. London and New York: Chapman and Hall, 1986.

[28] B. J. Zwolinski, et al., "Selected values of properties of hydrocarbons and related compounds," American Petroleum Institute Research Project 44, Thermodynamic Research Center, Texas A\&M University, 1977. 